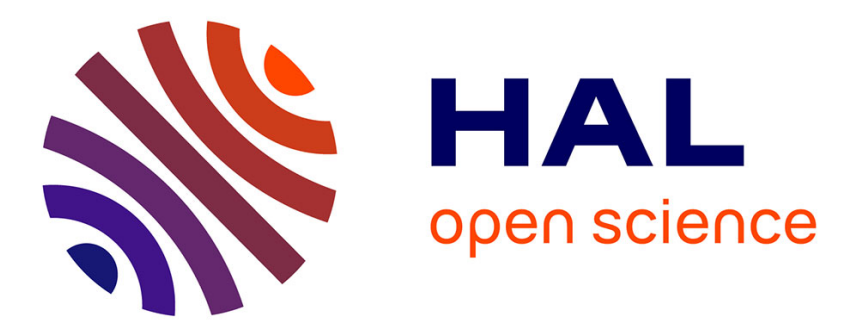

\title{
A phase-field approximation of the Steiner problem in dimension two
}

\author{
Antonin Chambolle, Luca Alberto Davide Ferrari, Benoît Merlet
}

\section{To cite this version:}

Antonin Chambolle, Luca Alberto Davide Ferrari, Benoît Merlet. A phase-field approximation of the Steiner problem in dimension two. Advances in Calculus of Variation, 2019, 12 (2), pp.157-179. 10.1515/acv-2016-0034. hal-01359483v2

\section{HAL Id: hal-01359483 \\ https://hal.science/hal-01359483v2}

Submitted on 27 Jun 2017

HAL is a multi-disciplinary open access archive for the deposit and dissemination of scientific research documents, whether they are published or not. The documents may come from teaching and research institutions in France or abroad, or from public or private research centers.
L'archive ouverte pluridisciplinaire HAL, est destinée au dépôt et à la diffusion de documents scientifiques de niveau recherche, publiés ou non, émanant des établissements d'enseignement et de recherche français ou étrangers, des laboratoires publics ou privés. 


\title{
A phase-field approximation of the Steiner problem in dimension two
}

\author{
A. Chambolle* $\quad$ L. Ferrari ${ }^{\dagger} \quad$ B. Merlet ${ }^{\ddagger}$
}

In this paper we consider the branched transportation problem in $2 \mathrm{D}$ associated with a cost per unit length of the form $1+\beta \theta$ where $\theta$ denotes the amount of transported mass and $\beta>0$ is a fixed parameter (notice that the limit case $\beta=0$ corresponds to the classical Steiner problem). Motivated by the numerical approximation of this problem, we introduce a family of functionals $\left(\left\{\mathcal{F}_{\varepsilon}\right\}_{\varepsilon>0}\right)$ which approximate the above branched transport energy. We justify rigorously the approximation by establishing the equicoercivity and the $\Gamma$-convergence of $\left\{\mathcal{F}_{\varepsilon}\right\}$ as $\varepsilon \downarrow 0$. Our functionals are modeled on the Ambrosio-Tortorelli functional and are easy to optimize in practice. We present numerical evidences of the efficiency of the method.

\section{Introduction}

In this paper, we introduce a phase-field approximation of a branched transportation energy for lines in the plane [6]. Our main goal is to derive a computationally tractable approximation of the Steiner problem (of minimizing the length of lines connecting a given set of points) in a phase-field setting. Similar results have been obtained recently by [8], however we believe our approach is slightly simpler and numerically easier to implement. We show that we can modify classical approximations for free discontinuity problems $[15$, $3,14,12]$ to address our specific problem, where the limiting energy is concentrated only on a singular one-dimensional network (and roughly measures its length). Numerical evidence illustrate the behaviour of these elliptic approximations. In this first study, we limit ourselves to the two-dimensional case, as in that case lines can locally be seen as discontinuities of piecewise constant functions, so that our construction can be derived in a quite simple way from the above mentioned previous works on free discontinuity problems. Higher dimension is more challenging from the topological point of view; an extension of this approach is currently in preparation.

${ }^{*}$ CNRS, CMAP, École Polytechnique CNRS UMR 7641, Route de Saclay, F-91128 Palaiseau Cedex France, email: antonin.chambolle@cmap.polytechnique.fr

${ }^{\dagger}$ CMAP, École Polytechnique, CNRS UMR 7641, Route de Saclay, F-91128 Palaiseau Cedex France, email: luca.ferrari@polytechnique.fr

${ }^{\ddagger}$ Laboratoire P. Painlevé, CNRS UMR 8524, Université Lille 1, F-59655 Villeneuve d'Ascq Cedex, France, email: benoit.merlet@math.univ-lille1.fr 
We now introduce precisely our mathematical framework. Let $\Omega \subset \mathbf{R}^{2}$ be a convex, bounded open set. We consider measures $\sigma \in \mathcal{M}\left(\bar{\Omega}, \mathbf{R}^{2}\right)$ of the form

$$
\sigma=\theta \xi \cdot \mathcal{H}^{1}\llcorner M
$$

where $M$ is a 1-dimensional rectifiable set oriented by a Borel measurable mapping $\xi$ : $M \rightarrow \mathbf{S}^{1}$ and $\theta: M \rightarrow \mathbf{R}_{+}$is a Borel measurable function representing the multiplicity. Such measure is called a rectifiable measure. We follow the notation of [16] and write $\sigma=U(M, \theta, \xi)$. Given a cost function $f \in C\left(\mathbf{R}_{+}, \mathbf{R}_{+}\right)$, we introduce the functional defined on $\mathcal{M}\left(\bar{\Omega}, \mathbf{R}^{2}\right) \rightarrow \mathbf{R}_{+} \cup\{+\infty\}$ as

$$
\mathcal{E}_{f}(\sigma):=\left\{\begin{array}{cl}
\int_{M} f(\theta) \mathrm{d} \mathcal{H}^{1} & \text { if } \sigma=U(\theta, \xi, M) \\
+\infty & \text { in the other cases. }
\end{array}\right.
$$

Given two probability measures $\omega_{1}$ and $\omega_{2}$ supported on a finite number of points in $\Omega$, denote with $S$ the union of their support, and consider the minimization of $\mathcal{E}_{f}(\sigma)$ for $\sigma \in \mathcal{M}\left(\bar{\Omega}, \mathbf{R}^{2}\right)$ satisfying the constraint

$$
\nabla \cdot \sigma=\omega_{1}-\omega_{2} \quad \text { in } \mathcal{D}^{\prime}\left(\mathbf{R}^{2}\right)
$$

In general, a model for branched transport connecting a set of sources $\left(\operatorname{supp}\left(\omega_{1}\right)\right)$ to a set of sinks $\left(\operatorname{supp}\left(\omega_{2}\right)\right)$ is obtained by choosing $f(\theta)=|\theta|^{\alpha}$ with $0 \leq \alpha \leq 1$ and minimizing the associated functional under a divergence constraint (1.1), as shown in [20]. In our result we obtain a linear combination depending on a parameter $\beta$ between the cost functions for $\alpha=0$ and $\alpha=1$, namely $f(\theta)=1+\beta|\theta|$. Such cost function for suitable choices of the constraint and the parameter $\beta$ gives a good approximation of the functional associated to the Steiner minimal tree problem. The direct numerical optimization of the functional $\mathcal{E}_{f}$ is not easy because we do not know a priori the topological properties of the tree $M$. For this reason it is interesting to optimize an "approximate" functional defined on more flexible objects such as functions. Such approximate model has been introduced in [16] where the authors study the $\Gamma$-convergence (see [10]) of a family of functionals inspired by the well known work of Modica and Mortola [15]. Another effort in this direction can be found in the work [8] where an approximation to the Steiner minimal tree problem ([2], [13] and [17]) is studied by means of analogous techniques.

Here, we consider variational approximations of some energies of the form $\mathcal{E}_{f}$ through a family of functionals modeled on the Ambrosio-Tortorelli functional [3]. To be more precise, we need to introduce some material. Let $\rho \in C_{c}^{\infty}\left(\mathbf{R}^{2}, \mathbf{R}_{+}\right)$be a classical radial mollifier with $\operatorname{supp} \rho \subset B_{1}(0)$ and $\int \rho=1$. For $\varepsilon \in(0,1]$, we set $\rho_{\varepsilon}(x)=\varepsilon^{-2} \rho\left(\varepsilon^{-1} x\right)$ and we define the space $V_{\varepsilon}(\Omega)$ of square integrable vector fields with weak divergence satisfying the constraint

$$
\nabla \cdot \sigma_{\varepsilon}=\left(\omega_{1}-\omega_{2}\right) * \rho_{\varepsilon} .
$$

For an $\eta=\eta(\varepsilon)>0$, we denote

$$
W_{\varepsilon}(\Omega)=\left\{\phi \in W^{1,2}(\Omega): \eta \leq \phi \leq 1 \text { in } \Omega, \phi \equiv 1 \text { on } \partial \Omega\right\} .
$$

Then we define the energy $\mathcal{F}_{\varepsilon}: \mathcal{M}\left(\Omega, \mathbf{R}^{2}\right) \times L^{1}(\Omega) \rightarrow[0,+\infty]$ as

$\mathcal{F}_{\varepsilon}(\sigma, \phi):= \begin{cases}\int_{\Omega} \frac{1}{2 \varepsilon} \phi^{2}|\sigma|^{2} \mathrm{~d} x+\int_{\Omega}\left[\frac{\varepsilon}{2}|\nabla \phi|^{2}+\frac{(1-\phi)^{2}}{2 \varepsilon}\right] \mathrm{d} x, & \text { if }(\sigma, \phi) \in V_{\varepsilon}(\Omega) \times W_{\varepsilon}(\Omega), \\ +\infty, & \text { in the other cases. }\end{cases}$ 
The first integral in the definition of the energy will be refered to as the "constraint component" while the second integral will be regarded as the "Modica-Mortola component". Let us briefly describe the qualitative properties of the associated minimization problem. First notice that the constraint (1.2) forces $\sigma$ to be supported on a set containing $S=\operatorname{supp}\left(\omega_{1}\right) \cup \operatorname{supp}\left(\omega_{2}\right)$. Next, the constraint component of the energy strongly penalizes $\phi^{2}|\sigma|^{2}$ so that $\phi$ should be small in the region where $|\sigma|$ is large. On the other hand the behavior of $\phi$ is controlled by the Modica-Mortola component that forces $\phi$ to be close to 1 a.e. in $\Omega$. Finally it has been proved that such component, as $\varepsilon$ converges to 0 , has a price proportional to the 1 -Hausdorff measure of the set $\{\phi \neq 1\}$. As a consequence, we expect the support of $\sigma$ and the energy to concentrate on paths joining the points in $\operatorname{supp}\left(\omega_{1}\right)$ with those in $\operatorname{supp}\left(\omega_{2}\right)$ similarly to [20]. The main part of the paper consists in making rigorous and quantitative this analysis.

From now on, we assume that there exists some $\beta \geq 0$ such that

$$
\frac{\eta}{\varepsilon} \stackrel{\varepsilon \downarrow 0}{\longrightarrow} \beta
$$

We denote $\mathcal{M}_{S}(\bar{\Omega})$ the set of $\mathbf{R}^{2}$-valued measures $\sigma \in \mathcal{M}\left(\mathbf{R}^{2}, \mathbf{R}^{2}\right)$ with support in $\bar{\Omega}$ such that the constaint (1.1) holds. We define the limit energy $\mathcal{E}_{\beta}: \mathcal{M}\left(\bar{\Omega}, \mathbf{R}^{2}\right) \times L^{1}(\Omega) \rightarrow$ $[0,+\infty]$ as

$$
\mathcal{E}_{\beta}(\sigma, \phi)=\left\{\begin{array}{cl}
\int_{M}(1+\beta \theta) \mathrm{d} \mathcal{H}^{1} & \text { if } \phi \equiv 1, \sigma \in \mathcal{M}_{S}(\bar{\Omega}) \text { and } \sigma=U(M, \theta, \xi) \\
+\infty & \text { in the other cases. }
\end{array}\right.
$$

We prove the $\Gamma$-convergence of the sequence $\left(\mathcal{F}_{\varepsilon}\right)$ to the energy $\mathcal{E}_{\beta}$ as $\varepsilon \downarrow 0$. More precisely the convergence holds in $\mathcal{M}\left(\bar{\Omega}, \mathbf{R}^{2}\right) \times L^{1}(\Omega)$ where $\mathcal{M}\left(\bar{\Omega}, \mathbf{R}^{2}\right)$ is endowed with the weak-* topology and $L^{1}(\Omega)$ is endowed with its classical strong topology.

We begin by proving the equicoercivity of the sequence $\left(\mathcal{F}_{\varepsilon}\right)$. In this statement and throughout the paper, we make a small abuse of language by denoting $\left(a_{\varepsilon}\right)_{\varepsilon \in(0,1]}$ and calling sequence a family $\left\{a_{\varepsilon}\right\}$ labeled by a continuous parameter $\varepsilon \in(0,1]$. In the same spirit, we call subsequence of $\left(a_{\varepsilon}\right)$, any sequence $\left(a_{\varepsilon_{j}}\right)$ with $\varepsilon_{j} \rightarrow 0$ as $j \rightarrow+\infty$.

We establish the following lower bound.

Theorem 1.1 ( $\Gamma-\lim$ inf $)$. For any sequence $\left(\sigma_{\varepsilon}, \phi_{\varepsilon}\right) \subset \mathcal{M}\left(\Omega, \mathbf{R}^{2}\right) \times L^{1}(\Omega)$ such that $\sigma_{\varepsilon} \stackrel{*}{\rightarrow} \sigma$ and $\phi_{\varepsilon} \rightarrow \phi$ in the $L^{1}(\Omega)$ topology, with $(\sigma, \phi) \in \mathcal{M}\left(\Omega, \mathbf{R}^{2}\right) \times L^{1}(\Omega)$

$$
\liminf _{k \rightarrow+\infty} \mathcal{F}_{\varepsilon}\left(\sigma_{\varepsilon}, \phi_{\varepsilon}\right) \geq \mathcal{E}_{\beta}(\sigma, \phi) .
$$

To complete the $\Gamma$-convergence analysis, we establish the matching $\Gamma$-limsup inequality.

Theorem $1.2(\Gamma-\limsup )$. For any $(\sigma, \phi) \subset \mathcal{M}\left(\Omega, \mathbf{R}^{2}\right) \times L^{1}(\Omega)$ there exists a sequence $\left(\sigma_{\varepsilon}, \phi_{\varepsilon}\right)$ such that $\sigma_{\varepsilon} \stackrel{*}{\rightarrow} \sigma$ and $\phi_{\varepsilon} \rightarrow \phi$ in the $L^{1}(\Omega)$ topology and

$$
\limsup _{k \rightarrow+\infty} \mathcal{F}_{\varepsilon}\left(\sigma_{\varepsilon}, \phi_{\varepsilon}\right) \leq \mathcal{E}_{\beta}(\sigma, \phi)
$$

Theorem 1.3 (Equicoercivity). Assume $\beta>0$. For any sequence $\left(\sigma_{\varepsilon}, \phi_{\varepsilon}\right)_{\varepsilon \in(0,1]} \subset$ $\mathcal{M}\left(\Omega, \mathbf{R}^{2}\right) \times L^{1}(\Omega)$ with uniformly bounded energies, i.e.

$$
\sup _{\varepsilon} \mathcal{F}_{\varepsilon}\left(\sigma_{\varepsilon}, \phi_{\varepsilon}\right)<+\infty
$$


there exist a subsequence $\varepsilon_{j} \downarrow 0$ and a measure $\sigma \in \mathcal{M}_{S}\left(\bar{\Omega}, \mathbf{R}^{2}\right)$ such that $\sigma_{\varepsilon_{j}} \rightarrow \sigma$ with respect to the weak-* convergence of measures and $\phi_{\varepsilon_{j}} \rightarrow 1$ in $L^{1}(\Omega)$. Moreover, $\sigma$ is a rectifiable measure (i.e. it is of the form $\sigma=U(M, \theta, \xi)$ ).

Remark 1 (Relation with the Steiner minimal tree problem). Let us discuss the relation between the limit functional and the Steiner minimal tree problem ([2], [13] and [17]). Indeed, given a set $S=\left\{x_{0}, \ldots, x_{N}\right\}$, the Steiner problem consists in finding the 1dimensional connected set of minimal length containing $S$. Let us associate the measures

$$
\omega_{1}=\delta_{x_{0}} \quad \text { and } \quad \omega_{2}=\frac{1}{N} \sum_{i=1}^{N} \delta_{x_{i}}
$$

to $S$. Then letting $\beta=0$ in the equation (1.5) leads to $\mathcal{E}_{0}(\sigma)=\mathcal{H}^{1}(\{x \in M: \theta(x)>0\})$ where $\sigma=U(M, \theta, \xi)$, and $S \subset \operatorname{supp}(\sigma)$ to fulfill the constraint (1.1). This is exactly the functional associated with the Steiner minimal tree problem. Let us stress out that the hypothesis $\beta>0$ is necessary to have compactness result since in Theorem 1.3, we show that

$$
\sup _{\varepsilon}\left|\sigma_{\varepsilon}\right|(\Omega) \leq \frac{C}{\beta}
$$

Nevertheless the $\Gamma$-convergence result still holds (without compactness) for $\beta=0$.

In the setting introduced in the previous Remark the functional defined in (1.3) is closely related to the one studied in [8], where the authors study the functional

$$
\frac{1}{c_{\varepsilon}} \sum_{1}^{N} d_{\phi}\left(x_{0}, x_{i}\right)+\int_{\Omega}\left[\frac{\varepsilon}{2}|\nabla \phi|^{2}+\frac{(1-\phi)^{2}}{2 \varepsilon}\right] \mathrm{d} x
$$

where

$$
d_{\phi}(x, y)=\inf \left\{\int_{\gamma} \phi(x) \mathcal{H}^{1}(x): \gamma \text { curve in } \Omega \text { connecting } x \text { and } y\right\} .
$$

The link with the present work lies in the relation,

$$
\sum_{i=1}^{N} d_{\phi}\left(x_{0}, x_{i}\right)=\min \left\{\int_{\Omega} \phi|\sigma| \mathrm{d} x: \nabla \cdot \sigma=\delta_{x_{0}}-\frac{1}{N} \sum_{i=1}^{N} \delta_{x_{i}}\right\} .
$$

Let us observe that by choosing as constraint component the term

$$
\int_{\Omega} \frac{\phi^{2}|\sigma|^{p}}{\varepsilon^{a}}
$$

for any $p>1$ we still would have obtained for $\Gamma$-limit the functional $\mathcal{E}_{\beta}$. In particular we have chosen a quadratic penalization $(p=2)$ on $\sigma$ since, in this way, we can solve the problem in sigma by solving a simple dual formulation, as shown in the Numerical Section. Finally let us point out that to ensure that the support of $\sigma$ connects the points in $S$ we need to privilege one point, namely $x_{0}$, among all the others. In our approach the defects introduced by the constraint affects only the mass component of the limit functional, which is controlled by the parameter $\beta$. Furthermore the family of functionals $\left\{\mathcal{E}_{\beta}\right\}, \Gamma$-converges to $\mathcal{E}_{0}$, as $\beta$ tends to zero. The latter allows to claim that for small values 
of $\beta$ and $\varepsilon$ the support of a minimizing $\sigma$ for the functional $\mathcal{F}_{\varepsilon}$ is a good approximation to a solution to the Steiner minimal tree problem. A different approach to this problem has been recently proposed in [7] where the authors study a phase-field approximation for rank one valued vector measures. The fact that we are working in dimension 2 is fundamental for the proof of Theorem 1.1 as it allows to locally rewrite the vector field $\sigma_{\varepsilon}$ as the rotated gradient of a function. We will study the higher dimensional cases in a forthcoming paper.

Structure of the paper: In Section 2 we introduce some notation and several tools and notions on $S B V$ functions and vector field measures. In Section 3 we study the behavior of the functional $\mathcal{F}_{\varepsilon}$ on vector fields of the form $\nabla u$ (dropping the divergence constraint). In Section 4 we prove the equicoercivity result, Theorem 1.3 and we establish the lower bound stated in Theorem 1.1. In Section 5 we prove the upper bound of Theorem 1.2. Finally, in the last section, we present and discuss various numerical simulations.

\section{Notation and Preliminary Results}

In the following $\Omega \subset \subset \hat{\Omega} \subset \mathbf{R}^{d}$ are bounded open convex sets. Given $X \subset \mathbf{R}^{d}$ (in practice $X=\Omega$ or $X=\hat{\Omega}$ ), we denote by $\mathcal{A}(X)$ the class of all relatively open subsets of $X$ and by $\mathcal{A}_{S}(X)$ the subclass of all simply connected relatively open sets $O \subset X$ such that $\bar{O} \cap S=\varnothing$. We denote by $\left(e_{1}, \ldots, e_{d}\right)$ the canonical orthonormal basis of $\mathbf{R}^{d}$, by $|\cdot|$ the euclidean norm and by $\langle\cdot, \cdot\rangle$ the euclidean scalar product in $\mathbf{R}^{d}$. The open ball of radius $r$ centered at $x \in \mathbf{R}^{d}$ is denoted by $B_{r}(x)$. The $(d-1)$-dimensional Hausdorff measure in $\mathbf{R}^{d}$ is denoted by $\mathcal{H}^{d-1}$. We write $|E|$ to denote the Lebesgue measure of a measurable set $E \subset \mathbf{R}^{d}$. When $\mu$ is a Borel meaure and $E \subset \mathbf{R}^{d}$ is a Borel set, we denote by $\mu\llcorner E$ the measure defined as $\mu\llcorner E(F)=\mu(E \cap F)$.

Let us remark that from Section 4 onwards, we work in dimension $d=2$.

For any fixed couple $(\sigma, \phi)$, with $\mathcal{F}_{\varepsilon}(\sigma, \phi ; O)$ we denote the value of the functional (1.3) on any set $O \in \mathcal{A}(\Omega)$. Similarly we define the with version $\mathcal{E}_{\beta}(\sigma, \phi ; O)$ the restriction of $\mathcal{E}_{\beta}$ to $O$.

\section{1. $\operatorname{BV}(\Omega)$ functions and Slicing}

$\operatorname{BV}(\Omega)$ is the space of functions $u \in L^{1}(\Omega)$ having as distributional derivative $D u$ a measure with finite total variation. Following the classical notation as in $[1,4]$ and $[9]$ for $u \in B V(\Omega)$ we have

$$
D u=\nabla u \mathrm{~d} x+\left(u^{+}-u^{-}\right) \nu_{u} \mathcal{H}^{d-1}\left\llcorner J_{u}+D^{c} u,\right.
$$

where $J_{u}$ is the set of "approximate jump points" $x$ where $y \mapsto u(x+\rho y)$ converge as $\rho \rightarrow 0$ to $u^{+} \chi_{\left\{y \cdot \nu_{u} \geq 0\right\}}+u^{-} \chi_{\left\{y \cdot \nu_{u}<0\right\}}$ for some $\left(u^{-}, u^{+}, \nu_{u}\right)$ and $D^{c} u$ is the Cantor "part". Let us introduce the space of special functions of bounded variation and a variant:

$$
\begin{gathered}
S B V(\Omega):=\left\{u \in B V(\Omega): D^{c} u=0\right\} \\
G S B V(\Omega):=\left\{u \in L^{1}(\Omega): \max (-T, \min (u, T)) \in S B V(\Omega) \forall T \in \mathbf{R}\right\} .
\end{gathered}
$$


Eventually, in Section 3, the following space of piecewise constant functions will be useful.

$$
P C(\Omega)=\{u \in G S B V(\Omega): \nabla u=0\} .
$$

To conclude this section we recall the slicing method for functions of bounded variation. Let $\xi \in \mathbf{S}^{d-1}$ and let

$$
\Pi_{\xi}:=\left\{y \in \mathbf{R}^{d}:\langle y, \xi\rangle=0\right\} .
$$

If $y \in \Pi_{\xi}$ and $E \subset \mathbf{R}^{d}$, we define the one dimensional slice

$$
E_{\xi, y}:=\{t \in \mathbf{R}: y+t \xi \in E\}
$$

For $u: \Omega \rightarrow \mathbf{R}$, we define $u_{\xi, y}: \Omega_{\xi, y} \rightarrow \mathbf{R}$ as

$$
u_{\xi, y}(t):=u(y+t \xi), \quad t \in \Omega_{\xi, y} .
$$

Functions in $G S B V(\Omega)$ can be characterized by one-dimensional slices (see [9, Thm. 4.1])

Theorem 2.1. Let $u \in G S B V(\Omega)$. Then for all $\xi \in \mathbf{S}^{d-1}$ we have

$$
u_{\xi, y} \in G S B V\left(\Omega_{\xi, y}\right) \quad \text { for } \mathcal{H}^{d-1} \text {-a.e. } y \in \Pi_{\xi} \text {. }
$$

Moreover for such $y$, we have

$$
\begin{gathered}
u_{\xi, y}^{\prime}(t)=\langle\nabla u(y+t \xi), \xi\rangle \quad \text { for a.e. } t \in \Omega_{\xi, y}, \\
J_{u_{\xi, y}}=\left\{t \in \mathbf{R}: y+t \xi \in J_{u}\right\}
\end{gathered}
$$

and

$$
u_{\xi, y}\left(t^{ \pm}\right)=u^{ \pm}(y+t \xi) \quad \text { or } \quad u_{\xi, y}\left(t^{ \pm}\right)=u^{\mp}(y+t \xi)
$$

according to whether $\left\langle\nu_{u}, \xi\right\rangle>0$ or $\left\langle\nu_{u}, \xi\right\rangle<0$. Finally, for every Borel function $g: \Omega \rightarrow$ $\mathbf{R}$,

$$
\int_{\Pi_{\xi}} \sum_{t \in J_{u_{\xi}, y}} g_{\xi, y}(t) \mathrm{d} \mathcal{H}^{d-1}(y)=\int_{J_{u}} g\left|\left\langle\nu_{u}, \xi\right\rangle\right| \mathrm{d} \mathcal{H}^{d-1} .
$$

Conversely if $u \in L^{1}(\Omega)$ and if for all $\xi \in\left\{e_{1}, \ldots, e_{d}\right\}$ and almost every $y \in \Pi_{\xi}$ we have $u_{\xi, y} \in S B V\left(\Omega_{\xi, y}\right)$ and

$$
\int_{\Pi_{\xi}}\left|D u_{\xi, y}\right|\left(\Omega_{\xi, y}\right) \mathrm{d} \mathcal{H}^{d-1}(y)<+\infty
$$

then $u \in S B V(\Omega)$.

\subsection{Rectifiable vector Measures}

Let us introduce the linear operator $\perp$ that associates to each vector $v=\left(v_{1}, v_{2}\right) \in \mathbf{R}^{2}$ the vector $v^{\perp}=\left(-v_{2}, v_{1}\right)$ obtained via a $90^{\circ}$ counterclockwise rotation of $v$. Notice that the $\perp$ operator maps divergence free $\mathbf{R}^{2}$-valued measures onto curl free $\mathbf{R}^{2}$-valued measures. Let $O \subset \mathbf{R}^{2}$ be a simply connected and bounded open set. It is possible to generalize Stokes Theorem to divergence free measures. If $\mu$ is a smooth divergence free vector field on $O$ we have $\mu=\nabla u^{\perp}$ for some smooth function with zero mean value. Then by Poincaré inequality $|u|_{L^{1}} \leq C|\mu|_{L^{1}}$. The result for $\mu$ general divergence free finite vector measure 
follows by regularization. On the other hand for $u \in P C(\Omega), \sigma:=D u^{\perp}$ is divergence free and,

$$
\sigma=\left(u^{+}-u^{-}\right) \nu_{u}^{\perp} \mathcal{H}^{1}=U\left(J_{u},[u], \nu_{u}^{\perp}\right) .
$$

Let us now produce an elementary example of measure $\gamma$ of the form $U(M, \theta, \xi)$.

Example 1. Given two points $x, y \in \Omega$ we consider a Lipschitz path $\psi(t)$ linking two points $x$ and $y$ in $\Omega$, parametrized on the interval $[0,1]$. We define the vector measure $\gamma \in \mathcal{M}\left(\Omega, \mathbf{R}^{2}\right)$ by

$$
(\phi, \gamma):=\int\langle\phi(\psi(t)), \dot{\psi}(t)\rangle \mathrm{d} t \quad \text { for any } \phi \in \mathcal{C}\left(\bar{\Omega}, \mathbf{R}^{2}\right) .
$$

We then have $\gamma=U(\psi([0,1]), 1, \dot{\psi})$. Notice that $\nabla \cdot \gamma=\delta_{x}-\delta_{y}$. In particular we denote with $[x, y]$ the Lipschitz path associated to the segment connecting the two points from $x$ to $y$, namely

$$
\psi(t)=(1-t) x+t y
$$

\section{Local Result}

In this section we introduce a localization of the family of functionals $\left(\mathcal{F}_{\varepsilon}\right)$ (see $(1.3)$ ). We establish a lower bound and a compactness property for these local energies.

Localization. Let $O \in A_{S}(\Omega)$ a simply connected relatively open subset of $\Omega$, for $u_{\varepsilon} \in$ $W^{1,2}(O)$ and $\phi_{\varepsilon} \in W^{1,2}(O)$, we define

$$
\mathcal{L F}_{\varepsilon}\left(u_{\varepsilon}, \phi_{\varepsilon} ; O\right):=\mathcal{F}_{\varepsilon}\left(\nabla u_{\varepsilon}, \phi_{\varepsilon} ; O\right)
$$

i.e. as the evaluation of the functional $\mathcal{F}$ on vector fields of the form $\nabla u$ with no requirement on the divergence. Notice that for $\varepsilon<d(O, S)$, we have $\nabla \cdot \sigma_{\varepsilon} \equiv 0$ in $O$ for any $\sigma_{\varepsilon} \in V_{\varepsilon}(\Omega)$. By Stokes theorem we have $D u_{\varepsilon}^{\perp}=\sigma_{\varepsilon}$ for some $u_{\varepsilon} \in W^{1,2}(O)$ and we have

$$
\mathcal{F}_{\varepsilon}\left(\sigma_{\varepsilon}, \phi_{\varepsilon} ; O\right)=\mathcal{L F}_{\varepsilon}\left(u_{\varepsilon}, \phi_{\varepsilon} ; O\right) .
$$

The rest of the section is devoted to the proof of

Theorem 3.1. Let $\left(u_{\varepsilon}\right)_{\varepsilon \in(0,1]} \subset W^{1,2}(O)$ be a family of functions with zero mean value and let $\left(\phi_{\varepsilon}\right) \subset W^{1,2}(O)$ such that $\phi_{\varepsilon} \in W^{1,2}(O,[\eta(\varepsilon), 1])$. Assume that $c_{0}:=\sup _{\varepsilon} \mathcal{L F}_{\varepsilon}\left(u_{\varepsilon}, \phi_{\varepsilon} ; O\right)$ is finite. Then there exist a subsequence $\varepsilon_{j}$ and a function $u \in B V(\Omega)$ such that

a) $\phi_{\varepsilon_{j}} \rightarrow 1$ in $L^{2}(O)$,

b) $u_{\varepsilon_{j}} \rightarrow u$ with respect to the weak-* convergence in $B V$,

c) $u \in P C(O)$.

Furthermore for any piecewise function $u \in P C(O)$ and any sequence $\left(u_{\varepsilon}, \phi_{\varepsilon}\right)$ such that $u_{\varepsilon} \stackrel{*}{\rightarrow} u$, we have the following lower bound of the energy:

$$
\liminf _{\varepsilon \rightarrow 0} \mathcal{L F}_{\varepsilon}\left(u_{\varepsilon}, \phi_{\varepsilon} ; O\right) \geq \int_{J_{u} \cap O}[1+\beta|[u]|] \mathrm{d} \mathcal{H}^{d-1}
$$


The proof is achieved in several steps and mostly follows ideas from [14] (see also [12]). In the first step we obtain (a) and (b). In step 2 we prove (c) and the lower bound for one dimensional slices of $\mathcal{L F}_{\varepsilon}$. Finally in step 3 we prove $(c)$ and the lower bound in dimension $d$. The construction of a recovery sequence that would complete the $\Gamma$-limit analysis is postponed to the global model in Section 5 .

Proof. Step 1: Item (a) is a straightforward consequence of the definition of the functional. Indeed, we have

$$
\int_{O}\left(1-\phi_{\varepsilon}\right)^{2} \mathrm{~d} x \leq \varepsilon \mathcal{L} \mathcal{F}_{\varepsilon}\left(\sigma_{\varepsilon}, \phi_{\varepsilon}\right) \leq c_{0} \varepsilon \stackrel{\varepsilon \downarrow 0}{\longrightarrow} 0 .
$$

For $(b)$, since $\left(u_{\varepsilon}\right)$ has zero mean value, we only need to show that $\sup _{\varepsilon \in(0,1]}\left|D u_{\varepsilon}\right|(O)<$ $+\infty$. Using Cauchy-Schwarz inequality we get

$$
\left[\left|D u_{\varepsilon}\right|(O)\right]^{2}=\left(\int_{O}\left|\nabla u_{\varepsilon}\right|\right)^{2} \leq\left(2 \varepsilon \int_{O} \frac{1}{\phi_{\varepsilon}^{2}}\right)\left(\frac{1}{\varepsilon} \int_{O} \phi_{\varepsilon}^{2}\left|\nabla u_{\varepsilon}\right|^{2}\right) .
$$

By assumption, the second therm in the right hand side of (3.1) is bounded by $2 c_{0}$. In order to estimate the first term we split $O$ in the two sets $\left\{\phi_{\varepsilon}<1 / 2\right\}$ and $\left\{\phi_{\varepsilon} \geq 1 / 2\right\}$. We have,

$$
2 \varepsilon \int_{O} \frac{1}{\phi_{\varepsilon}^{2}}=2 \varepsilon\left(\int_{\left\{\phi_{\varepsilon}<1 / 2\right\}} \frac{1}{\phi_{\varepsilon}^{2}}+\int_{\left\{\phi_{\varepsilon} \geq 1 / 2\right\}} \frac{1}{\phi_{\varepsilon}^{2}}\right) .
$$

Since $\eta \leq \phi_{\varepsilon} \leq 1 / 2$ on $\left\{\phi_{\varepsilon} \leq 1 / 2\right\}$ it holds $\phi_{\varepsilon}^{2}\left(1-\phi_{\varepsilon}\right)^{2} \geq \eta^{2}(1-1 / 2)^{2}$ therefore

$$
\begin{aligned}
\int_{\left\{\phi_{\varepsilon}<1 / 2\right\}} \frac{1}{\phi_{\varepsilon}^{2}} & \leq \frac{2 \varepsilon}{\eta^{2}(1-1 / 2)^{2}} \int_{\left\{\phi_{\varepsilon}<1 / 2\right\}} \frac{\left(1-\phi_{\varepsilon}\right)^{2}}{2 \varepsilon} \leq \frac{8 \varepsilon}{\eta^{2}} c_{0}, \\
\int_{\left\{\phi_{\varepsilon} \geq 1 / 2\right\}} \frac{1}{\phi_{\varepsilon}^{2}} & \leq \int_{\left\{\phi_{\varepsilon} \geq 1 / 2\right\}} \frac{1}{(1 / 2)^{2}}=4\left|\left\{\phi_{\varepsilon} \geq 1 / 2\right\}\right| .
\end{aligned}
$$

Eventually, as $\left|\left\{\phi_{\varepsilon} \geq 1 / 2\right\}\right| \leq|O|$, combining these estimates with (3.1) we obtain

$$
\left[\left|D u_{\varepsilon}\right|(O)\right]^{2} \leq \frac{\varepsilon^{2}}{\eta^{2}} 16 c_{0}^{2}+8 \varepsilon|O| c_{0} \stackrel{\varepsilon \downarrow 0}{\longrightarrow} \frac{16 c_{0}^{2}}{\beta^{2}}<\infty .
$$

This establishes (b).

Step 2: In this step we suppose $O$ to be an interval of $\mathbf{R}$, so that $u_{\varepsilon}, \phi_{\varepsilon}$ are onedimensional. We first prove that $u$ is piecewise constant. The idea is that in view of the constraint component of the energy, variations of $u_{\varepsilon}$ are balanced by low values of $\phi_{\varepsilon}$. On the other hand the Modica-Mortola component of the energy implies that $\phi_{\varepsilon} \simeq 1$ in most of the domain and that transitions from $\phi_{\varepsilon} \simeq 1$ to $\phi_{\varepsilon} \simeq 0$ have a constant positive cost (and therefore can occur only finitely many times).

Step 2.1: (Proof of $u \in P C(O)$.) Let us define

$$
B_{\varepsilon}:=\left\{x \in O: \phi_{\varepsilon}(x)<\frac{3}{4}\right\} \supset A_{\varepsilon}:=\left\{x \in O: \phi_{\varepsilon}(x)<\frac{1}{2}\right\},
$$

and let

$$
C_{\varepsilon}=\left\{I \text { connected component of } B_{\varepsilon}: I \cap A_{\varepsilon} \neq \varnothing\right\}
$$


Let us show that the cardinality of $C_{\varepsilon}$ is bounded by a constant independent of $\varepsilon$. Let $\varepsilon$ be fixed and consider an interval $I \in C_{\varepsilon}$. Let $a, b \in \bar{I}$ such that $\left\{\phi_{\varepsilon}(a), \phi_{\varepsilon}(b)\right\}=\{1 / 2,3 / 4\}$. Using the usual Modica-Mortola trick, we have

$\mathcal{L F}_{\varepsilon}\left(u_{\varepsilon}, \phi_{\varepsilon} ; I\right) \geq \int_{I} \varepsilon\left|\phi_{\varepsilon}^{\prime}\right|^{2}+\frac{\left(1-\phi_{\varepsilon}\right)^{2}}{4 \varepsilon} \mathrm{d} x \geq \int_{(a, b)}\left|\phi_{\varepsilon}^{\prime}\right|\left(1-\phi_{\varepsilon}\right) \mathrm{d} x \geq \int_{1 / 2}^{3 / 4}(1-t) d t=\frac{3}{2^{5}}$.

Since all the elements of $C_{\varepsilon}$ are disjoint and $\mathcal{L F}_{\varepsilon}\left(u_{\varepsilon}, \phi_{\varepsilon}, \cdot\right)$ is additive, we deduce from the energy bound that

$$
\# C_{\varepsilon} \leq 2^{5} c_{0} / 3
$$

where we denote $\# C_{\varepsilon}$ the cardinality of $C_{\varepsilon}$. Next, up to extracting a subsequence we assume that $\# C_{\varepsilon}=N$ is fixed. The elements of $C_{\varepsilon}$ are written on the form $I_{i}^{\varepsilon}=$ $\left(m_{i}^{\varepsilon}-w_{i}^{\varepsilon}, m_{i}^{\varepsilon}+w_{i}^{\varepsilon}\right)$ for $i=1, \cdots, N$ with $m_{i}^{\varepsilon}<m_{i+1}^{\varepsilon}$. Since $\phi_{\varepsilon} \rightarrow 1$ in $L^{1}(O)$ we have

$$
\sum_{I_{i}^{\varepsilon} \in C_{\varepsilon}}\left|I_{i}^{\varepsilon}\right|=\sum_{i} 2 w_{i}^{\varepsilon} \rightarrow 0
$$

Up to extracting a subsequence, we can assume that each sequence $\left(m_{i}^{\varepsilon}\right)$ converges in $\bar{O}$. We call $m_{1} \leq m_{2} \leq \cdots \leq m_{N}$ their respective limits. We now prove that

$$
|D u|\left(O \backslash\left\{m_{i}\right\}_{i=1}^{N}\right)=0,
$$

thus $\operatorname{supp}(|D u|) \subset\left\{m_{1}, \cdots, m_{N}\right\}$. The latter ensures that $u$ has no Cantor component since $D u$ is supported on a finite number of points and that is a.e. constant outside $\left\{m_{1}, \cdots, m_{N}\right\}$ so that $u \in P C(O),(2.1)$. To this aim, we fix $x \in O \backslash\left\{m_{i}\right\}_{i=0}^{N}$ and establish the existence of a neighborhood $B_{\delta}(x)$ of $x$ for which $|D u|\left(B_{\delta}(x)\right)=0$. Let $0<\delta \leq \min _{i}\left|x-m_{i}\right| / 2$. Equation (3.5) ensures that for $\varepsilon \operatorname{small}$ enough $B_{\delta}(x) \cap C_{\varepsilon}=\varnothing$. Notice that from the definitions in (3.3) and (3.4) we have that $\phi_{\varepsilon} \geq 1 / 2$ outside the union of the sets in $C_{\varepsilon}$. Hence, using Cauchy-Scwarz inequality, we have for $\varepsilon$ small enough,

$$
\left(\int_{B_{\delta}(x)}\left|u_{\varepsilon}^{\prime}\right| \mathrm{d} x\right)^{2} \leq 2 \delta \int_{B_{\delta}(x)}\left|u_{\varepsilon}^{\prime}\right|^{2} \mathrm{~d} x \leq(2 \delta)(2 \varepsilon) 4\left(\frac{1}{2 \varepsilon} \int_{B_{\delta}(x)} \phi_{\varepsilon}^{2}\left|u_{\varepsilon}^{\prime}\right|^{2} \mathrm{~d} x\right) \leq 16 c_{0} \varepsilon \delta \stackrel{\varepsilon \downarrow 0}{\longrightarrow} 0 .
$$

By lower semicontinuity of the total variation on open sets we conclude that $|D u|\left(B_{\delta}(x)\right)=$ 0 , which proves the claim (3.6).

Step 2.2: (Proof of the lower bound for $\mathcal{L F}_{\varepsilon}$.) Without loss of generality we can assume $N=1$, thus $J_{u}$ is composed of a single point, otherwise the argument we propose can be applied on each $m_{i}$ separately. Up to a translation $m_{1}=0$ and we denote $D:=u\left(0^{+}\right)=$ $-u\left(0^{-}\right)>0$. For any $0<d<D$ there exist six points $y_{1}<x_{\varepsilon}^{1} \leq \tilde{x}_{\varepsilon}^{1}<\tilde{x}_{\varepsilon}^{2} \leq x_{\varepsilon}^{2}<y_{2}$ such that

$$
\begin{gathered}
\lim _{\varepsilon \rightarrow 0} \phi_{\varepsilon}\left(y_{1}\right)=\lim _{\varepsilon \rightarrow 0} \phi_{\varepsilon}\left(y_{2}\right)=1, \\
\lim _{\varepsilon \rightarrow 0} \phi_{\varepsilon}\left(x_{\varepsilon}^{1}\right)=\lim _{\varepsilon \rightarrow 0} \phi_{\varepsilon}\left(x_{\varepsilon}^{2}\right)=0, \\
u_{\varepsilon}\left(\tilde{x}_{\varepsilon}^{1}\right)=-D+d, \quad u_{\varepsilon}\left(\tilde{x}_{\varepsilon}^{2}\right)=D-d .
\end{gathered}
$$

Since $\phi_{\varepsilon} \rightarrow \phi$ and $u_{\varepsilon} \rightarrow u$ in $L^{1}$ up to a subsequence they converge point-wise almost everywhere and this implies the first and third fact. Let $\inf _{\left(y_{1}, y_{2}\right)} \phi_{\varepsilon}=c_{\varepsilon}$, then Jensen inequality implies

$$
c_{0} \geq \int_{y_{1}}^{y_{2}} \frac{\phi_{\varepsilon}^{2}\left|u_{\varepsilon}^{\prime}\right|^{2}}{2 \varepsilon} \mathrm{d} x \geq \frac{c^{2}}{2 \varepsilon\left(y_{2}-y_{1}\right)}\left(\int_{y_{1}}^{y_{2}}\left|u_{\varepsilon}^{\prime}\right| \mathrm{d} x\right)^{2} .
$$


Then $c_{\varepsilon}$ must vanish with $\varepsilon$ implying statement (3.7). Using the Modica-Mortola trick in the intervals $\left(y_{1}, x_{\varepsilon}^{1}\right)$ and $\left(x_{\varepsilon}^{2}, y_{2}\right)$ as above, we compute:

$\liminf _{\varepsilon \downarrow 0} \mathcal{L F}_{\varepsilon}\left(u_{\varepsilon}, \phi_{\varepsilon} ;\left(y_{1}, x_{\varepsilon}^{1}\right) \cup\left(x_{\varepsilon}^{2}, y_{2}\right)\right) \geq \liminf _{\varepsilon \downarrow 0} \int_{y_{1}}^{x_{\varepsilon}^{1}}\left(1-\phi_{\varepsilon}\right)\left|\phi_{\varepsilon}^{\prime}\right| \mathrm{d} x+\int_{x_{\varepsilon}^{2}}^{y_{2}}\left(1-\phi_{\varepsilon}\right)\left|\phi_{\varepsilon}^{\prime}\right| \mathrm{d} x \geq 1$.

For the estimate on the interval $I_{\varepsilon}=\left(\tilde{x}_{\varepsilon}^{1}, \tilde{x}_{\varepsilon}^{2}\right)$ let us introduce:

$$
\begin{gathered}
G_{\varepsilon}:=\left\{w \in W^{1,2}\left(I_{\varepsilon}\right): w\left(\tilde{x}_{\varepsilon}^{1}\right)=-D+d, w\left(\tilde{x}_{\varepsilon}^{2}\right)=D-d\right\}, \\
Z_{\varepsilon}:=\left\{z \in W^{1,2}\left(I_{\varepsilon}\right): \eta \leq z \leq 1 \text { a.e. on } I_{\varepsilon}\right\}, \\
H_{\varepsilon}(w, z):=\int_{I_{\varepsilon}}\left(\frac{1}{2 \varepsilon} z^{2}\left|w^{\prime}\right|^{2}+\frac{(1-z)^{2}}{2 \varepsilon}\right) \mathrm{d} x, \\
h_{\varepsilon}(z)=\inf _{w \in W_{\varepsilon}} H_{\varepsilon}(w, z) \text { for } z \in Z_{\varepsilon} .
\end{gathered}
$$

Note that for $w \in G_{\varepsilon}$ and $z \in Z_{\varepsilon}$, we can apply an inequality similar to (3.1). In particular, for $z$ replacing $\phi_{\varepsilon}$ and $w^{\prime}$ taking the place of $D u$ we get

$$
\left(\int_{I_{\varepsilon}}\left|w^{\prime}\right| \mathrm{d} x\right)^{2} \leq\left(\int_{I_{\varepsilon}} z^{2}\left|w^{\prime}\right|^{2}\right)\left(\int_{I_{\varepsilon}} \frac{1}{z^{2}}\right)
$$

Reversing the latter and taking into account the conditions on $w$ obtains

$$
\int_{I_{\varepsilon}} z^{2}\left|w^{\prime}\right|^{2} \geq\left(\int_{I_{\varepsilon}}\left|w^{\prime}\right| \mathrm{d} x\right)^{2}\left(\int_{I_{\varepsilon}} \frac{1}{z^{2}}\right)^{-1} \geq 4(D-d)^{2}\left(\int_{I_{\varepsilon}} \frac{1}{z^{2}}\right)^{-1} .
$$

From this we deduce the lower bound

$$
h_{\varepsilon}(z) \geq 4(D-d)^{2}\left(2 \varepsilon \int_{I_{\varepsilon}} \frac{1}{z^{2}} \mathrm{~d} x\right)^{-1}+\int_{I_{\varepsilon}}\left(\frac{(1-z)^{2}}{2 \varepsilon}\right) \mathrm{d} x .
$$

Let us remark that optimizing $H_{\varepsilon}(w, z)$ with respect to $w \in G_{\varepsilon}$ we see that this inequality is actually an equality.

Consider for $0<\lambda<1$ the inequalities:

$$
\int_{\left\{x \in I_{\varepsilon}: \phi_{\varepsilon} \geq \lambda\right\}} \frac{1}{\phi_{\varepsilon}^{2}} \leq \frac{\mathcal{L}^{1}\left(I_{\varepsilon}\right)}{\lambda^{2}} \quad \text { and } \quad \int_{\left\{x \in I_{\varepsilon}: \phi_{\varepsilon}<\lambda\right\}} \frac{1}{\phi_{\varepsilon}^{2}} \leq \frac{1}{(1-\lambda)^{2}} \frac{2 \varepsilon}{\eta^{2}}\left(\int_{I_{\varepsilon}} \frac{\left(1-\phi_{\varepsilon}\right)^{2}}{2 \varepsilon} \mathrm{d} x\right) \text {. }
$$

Applying both of them in (3.9) we obtain

$$
\begin{aligned}
\mathcal{L F}_{\varepsilon}\left(u_{\varepsilon}, \phi_{\varepsilon}, I_{\varepsilon}\right) & \geq h_{\varepsilon}\left(\phi_{\varepsilon}\right) \\
& \geq \frac{2(D-d)^{2}}{\frac{\varepsilon \mathcal{L}^{1}\left(I_{\varepsilon}\right)}{\lambda^{2}}+\frac{1}{(1-\lambda)^{2}} \frac{2 \varepsilon^{2}}{\eta^{2}}\left(\int_{I_{\varepsilon}} \frac{\left(1-\phi_{\varepsilon}\right)^{2}}{2 \varepsilon} \mathrm{d} x\right)}+\int_{I_{\varepsilon}}\left(\frac{\left(1-\phi_{\varepsilon}\right)^{2}}{2 \varepsilon}\right) \mathrm{d} x \\
& \geq 2(1-\lambda) \frac{\eta}{\varepsilon}(D-d)-(1-\lambda)^{2} \frac{\eta^{2}}{2 \varepsilon} \frac{\mathcal{L}^{1}\left(I_{\varepsilon}\right)}{\lambda^{2}}
\end{aligned}
$$

where the latter inequality is obtained by minimizing the function:

$$
t \mapsto \frac{2(D-d)^{2}}{\frac{\varepsilon \mathcal{L}^{1}\left(I_{\varepsilon}\right)}{\lambda^{2}}+\frac{1}{(1-\lambda)^{2}} \frac{2 \varepsilon^{2}}{\eta^{2}} t}+t
$$


Therefore we can pass to the limit in (3.10) and obtain:

$$
\liminf _{\varepsilon \downarrow 0} \mathcal{L F}_{\varepsilon}\left(u_{\varepsilon}, \phi_{\varepsilon}, I_{\varepsilon}\right) \geq(1-\lambda) \beta 2(D-d) .
$$

Sending $\lambda$ and $d$ to 0 and recalling the estimate in (3.8) we get

$$
\liminf _{\varepsilon \downarrow 0} \mathcal{L F}_{\varepsilon}\left(u_{\varepsilon}, \phi_{\varepsilon},\left(y_{1}, y_{2}\right)\right) \geq 1+\beta 2 D=1+\beta\left|u\left(0^{+}\right)-u\left(0^{-}\right)\right| .
$$

Step 3: Indeed by Fatou's Lemma for any $\xi \in \mathbf{S}^{d-1}$ and $\mathcal{H}^{d-1}$ almost every $y \in \Omega_{\xi}$ it holds

$$
\begin{aligned}
\liminf _{\varepsilon \downarrow 0} \mathcal{L F}_{\varepsilon}\left(u_{\varepsilon}, \phi_{\varepsilon} ; O\right) & \geq \\
\int_{\Pi_{\xi}} \liminf _{\varepsilon \downarrow 0} & \left(\int_{O_{y}^{\xi}} \frac{1}{2 \varepsilon}\left(\phi_{\varepsilon}^{2}\right)_{y}^{\xi}\left|\left(u_{\varepsilon}^{\prime}\right)_{y}^{\xi}\right|^{2}+\frac{\varepsilon}{2}\left|\left(\phi_{\varepsilon}^{\prime}\right)_{y}^{\xi}\right|^{2}+\frac{\left(1-\left(\phi_{\varepsilon}\right)_{y}^{\xi}\right)^{2}}{2 \varepsilon} \mathrm{d} t\right) \mathrm{d} \mathcal{H}^{d-1}(y) .
\end{aligned}
$$

Then by the results in Step 2.1 and 2.2, in particular inequality (3.11), it holds

$$
\liminf _{\varepsilon \downarrow 0} \mathcal{L F}_{\varepsilon}\left(u_{\varepsilon}, \phi_{\varepsilon} ; O\right) \geq \int_{\Pi_{\xi}} \sum_{m_{i} \in\left(J_{u}\right)_{y}^{\xi}}\left[1+\beta\left|u_{y}^{\xi}\left(m_{i}^{+}\right)-u_{y}^{\xi}\left(m_{i}^{-}\right)\right|\right] \mathrm{d} \mathcal{H}^{d-1}(y) .
$$

Therefore by Theorem 2.1 we have $u \in S B V(O)$. Moreover, since $\left(u^{\prime}\right)_{y}^{\xi}=0$ on each slice, we have $u \in P C(O)$. Applying identity (2.2) we get

$$
\liminf _{\varepsilon \rightarrow 0} \mathcal{L F}_{\varepsilon}\left(u_{\varepsilon}, \phi_{\varepsilon} ; O\right) \geq \int_{J_{u} \cap O}\left|\nu_{u} \cdot \xi\right|[1+\beta|[u]|] \mathrm{d} \mathcal{H}^{d-1} .
$$

In order to conclude, we use the following localization method stated by Braides in $[9$, Prop. 1.16].

Lemma 3.1. Let $\mu: \mathcal{A}(X) \rightarrow[0,+\infty)$ be an open-set function superadditive on open sets with disjoint compact closures and let $\lambda$ be a positive measure on $X$. For any $i \in \mathbf{N}$ let $\psi_{i}$ be a Borel function on $X$ such that $\mu(A) \geq \int_{A} \psi_{i} \mathrm{~d} \lambda$ for all $A \in \mathcal{A}(X)$. Then

$$
\mu(A) \geq \int_{A} \psi \mathrm{d} \lambda
$$

where $\psi:=\sup _{i} \psi_{i}$.

For any $u \in P C(O)$ let us introduce the increasing set function $\mu$ defined on $\mathcal{A}(O)$ by

$$
\mu(A):=\inf _{\phi_{\varepsilon} \rightarrow 1, u_{\varepsilon}{ }^{*} u}\left\{\liminf _{\varepsilon \rightarrow 0} \mathcal{L} \mathcal{F}_{\varepsilon}\left(u_{\varepsilon}, \phi_{\varepsilon} ; A\right)\right\}, \quad \text { for any } A \in \mathcal{A}(O) .
$$

Observe that for any two open sets $A$ and $B$ with disjoint compact closure and for any $\left(u_{\varepsilon}, \phi_{\varepsilon}\right)$ such that $u_{\varepsilon} \stackrel{*}{\rightarrow} u$ and $\phi_{\varepsilon} \rightarrow 1$ on $A \cup B$, the restriction of $u_{\varepsilon}$ to $A$ (resp. $B$ ) weak-* converges in $A$ (resp. $B$ ) to the restriction of $u$ on $A$ (resp. $B$ ) and it follows

$$
\mu(A+B) \geq \mu(A)+\mu(B) .
$$

This proves that $\mu$ is superaddittive on open sets with disjoint compact closures. Let $\lambda$ be a Radon measure defined as

$$
\lambda:=\left[1+\beta\left|u\left(x^{+}\right)-u\left(x^{-}\right)\right|\right] \mathcal{H}^{d-1}\left\llcorner J_{u} .\right.
$$


Fix a sequence $\left(\xi_{i}\right)_{i \in \mathbf{N}}$ dense in $\mathbf{S}^{d-1}$. By (3.12) we have

$$
\mu(O) \geq \int_{O} \psi_{i} \mathrm{~d} \lambda, \quad i \in \mathbf{N}
$$

where

$$
\psi_{i}(x):= \begin{cases}\left|\left\langle\nu_{u}(x), \xi_{i}\right\rangle\right|, & \text { if } x \in J_{u} \\ 0, & \text { if } x \in O \backslash J_{u} .\end{cases}
$$

Hence by Lemma 3.1 we finally obtain

$$
\liminf _{\varepsilon \rightarrow 0} \mathcal{L F}_{\varepsilon}\left(u_{\varepsilon}, \phi_{\varepsilon} ; O\right) \geq \int_{O} \sup _{i} \psi_{i}(x) \mathrm{d} \mu=\int_{J_{u} \cap O}[1+\beta|[u]|] \mathrm{d} \mathcal{H}^{d-1} .
$$

\section{Equicoercivity and $\Gamma$-liminf}

Let us first produce the following construction.

Lemma 4.1. Given two probability measures $\omega_{1}$ and $\omega_{2}$ supported on a finite set of points $S=\left\{x_{0}, \ldots, x_{N}\right\}$, there exists a vector measure $\gamma=U\left(M_{\gamma}, \theta_{\gamma}, \xi_{\gamma}\right)$ and a finite partition $\left(\Omega_{i}\right) \subset \mathcal{A}(\Omega)$ of $\Omega$ such that

a) $\nabla \cdot \gamma=-\omega_{1}+\omega_{2}$,

b) each $\Omega_{i}$ is a polyhedron,

c) $M_{\gamma} \subset \bigcup_{i} \partial \Omega_{i}$

d) $\Omega_{i}$ is of finite perimeter for each $i$ and $\Omega_{i} \cap \Omega_{j}=\emptyset$ for $i \neq j$,

e) $\mathcal{L}^{2}\left(\Omega \backslash \cup_{i} \Omega_{i}\right)=0$.

Moreover if $M$ is a 1 dimensional countably rectifiable set, we can choose $\gamma$ and $\left(\Omega_{i}\right)$ such that $\mathcal{H}^{1}\left(M \cap \bigcup_{i} \partial \Omega_{i}\right)=0$.

Proof. Let us fix a point $p \in \Omega \backslash S$ and assume

$$
\omega_{1}-\omega_{2}=\sum_{i=0}^{N} a_{i} \delta_{x_{i}}
$$

Following the Example 1 we can construct a measure $\gamma_{i}$ supported on the segment $\left[p, x_{i}\right]$ such that $\nabla \cdot \gamma_{i}=\delta_{x_{i}}-\delta_{p}$ for $i \in\{0, \cdots, N\}$. We define

$$
\gamma=\sum_{i=1}^{N} a_{i} \gamma_{i}
$$

By construction $(a)$ holds true. Moreover, up to a small shift of $p$ we may assume that $\left[p, x_{i}\right] \cap\left[p, x_{j}\right]=\{p\}$ for $i \neq j$.

Next, let $D_{j}$ be the straight line supporting $\left[p, x_{j}\right]$. We define the sets $\left(\Omega_{i}\right)$ as the connected components of $\Omega \backslash\left(D_{0} \cup \cdots \cup D_{N}\right)$. We see that $(c, d, e)$ hold true.

For the last statement, we observe that by the coarea formula, we have $\mathcal{H}^{1}\left(M \cap \bigcup_{i} \partial \Omega_{i}\right)=0$ for a.e. choice of $p$. 


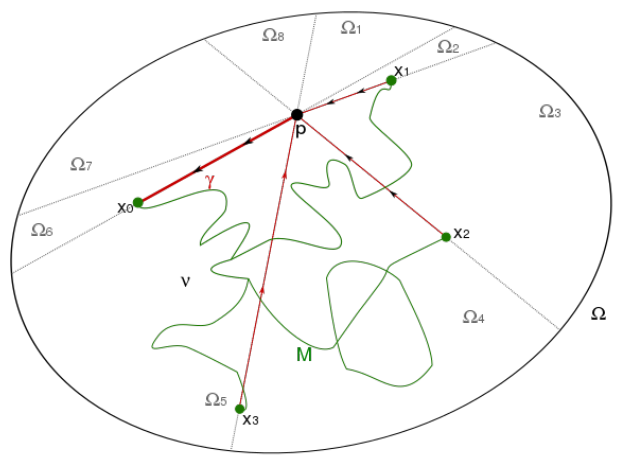

Figure 1: Example of the construction of the $\mathcal{H}^{1}$-rectifiable measure $\gamma(\mathrm{red})$ and of the partition $\left\{\Omega_{i}\right\}$ (gray) in the case $M$ (green) is being a $\mathcal{H}^{1}$-rectifiable set. Here $\omega_{1}=\delta_{x_{0}}$ and $\omega_{2}=1 / 3\left(\delta_{x_{1}}+\delta_{x_{2}}+\delta_{x_{3}}\right)$.

We now prove the compactness property (Theorem 1.3). Let us consider a sequence $\left(\sigma_{\varepsilon}, \phi_{\varepsilon}\right) \in \mathcal{M}\left(\Omega, \mathbf{R}^{2}\right)$ uniformly bounded in energy by $c_{0}<+\infty$,

$$
0 \leq \mathcal{F}_{\varepsilon}\left(\sigma_{\varepsilon}, \phi_{\varepsilon}\right) \leq c_{0} \quad \text { for } \varepsilon \in(0,1]
$$

Proof of Theorem 1.3. First observe that by definition (1.3) and equation (4.1), we have $\sigma_{\varepsilon} \in V_{\varepsilon}(\Omega)$ and $\phi_{\varepsilon} \in W_{\varepsilon}(\Omega)$. Next, using the arguments of Step 1 of the proof of Theorem 3.1, with $\left|\sigma_{\varepsilon}\right|$ instead of $\left|\nabla u_{\varepsilon}\right|$, inequality (3.2) reads

$$
\left|\sigma_{\varepsilon}\right|(\Omega) \leq \sqrt{16 \frac{\varepsilon^{2}}{\eta^{2}} c_{0}^{2}+8 \varepsilon|\Omega| c_{0}} \stackrel{\varepsilon \downarrow 0}{\longrightarrow} \frac{4 c_{0}}{\beta}<\infty .
$$

Thus the total variation of $\left(\sigma_{\varepsilon}\right)_{\varepsilon}$ is uniformly bounded as long as $\beta>0$ and there exists a $\sigma \in \mathcal{M}_{S}(\bar{\Omega})$ such that up to a subsequence $\sigma_{\varepsilon} \stackrel{*}{\rightarrow} \sigma$ in $\mathcal{M}(\bar{\Omega})$. Now, considering the last term in the energy (1.3) we have

$$
\int_{\Omega}\left(1-\phi_{\varepsilon}\right)^{2} \mathrm{~d} x \leq 2 \varepsilon \mathcal{F}_{\varepsilon}\left(\sigma_{\varepsilon}, \phi_{\varepsilon}\right) \leq 2 \varepsilon c_{0} \rightarrow 0
$$

Hence, $\phi_{\varepsilon} \rightarrow 1$ in $L^{2}(\Omega)$. 
Let us now study the structure of the limit measure $\sigma$. Let us recall that $\hat{\Omega}$ is a bounded convex relatively open set such that $\bar{\Omega} \subset \hat{\Omega}$ and let us extend $\sigma_{\varepsilon}$ by 0 and $\phi_{\varepsilon}$ by 1 in $\hat{\Omega} \backslash \bar{\Omega}$. Obviously we have $\mathcal{F}_{\varepsilon}\left(\sigma_{\varepsilon}, \phi_{\varepsilon} ; \hat{\Omega}\right)=\mathcal{F}_{\varepsilon}\left(\sigma_{\varepsilon}, \phi_{\varepsilon} ; \Omega\right)$, therefore for any $O \in \mathcal{A}_{s}(\hat{\Omega})$ applying the localization described in Section 3 we can associate to each $\sigma_{\varepsilon}$ a function $u_{\varepsilon} \in W^{1,2}(O)$ with mean value 0 such that $\sigma_{\varepsilon}=\nabla u_{\varepsilon}^{\perp}$ in $O$. Since $\left|\nabla u_{\varepsilon}^{\perp}\right|=\left|\nabla u_{\varepsilon}\right|$ by Theorem 3.1 there exists a $u \in P C(O)$ such that, up to extracting a subsequence, $u_{\varepsilon} \stackrel{*}{\rightarrow} u$. Eventually, from formula (2.3), we get

$$
\sigma\left\llcorner O=D u^{\perp}\left\llcorner O=-[u] \nu_{J_{u}}^{\perp} \mathcal{H}^{1}\left\llcorner\left(J_{u} \cap O\right) .\right.\right.\right.
$$

Since we can cover $\bar{\Omega} \backslash S$ by countable many sets $O \in \mathcal{A}_{S}(\hat{\Omega})$, this shows that $\sigma$ decomposes as

$$
\sigma=U\left(M_{\sigma}, \theta_{\sigma}, \xi_{\sigma}\right)+\mu
$$

Where $\mu$ is a measure absolutely continuous with respect to $\mathcal{H}^{0}\llcorner S$. By Lemma 4.1 there exists a rectifiable measure $\gamma=U\left(M_{\gamma}, \theta_{\gamma}, \xi_{\gamma}\right)$ such that $\nabla \cdot(\sigma+\gamma)=0$ and $\mathcal{H}^{1}\left(M_{\gamma} \cap M_{\sigma}\right)=$ 0 . Then there exists a $u \in B V(\Omega)$ such that $D u=\sigma^{\perp}+\gamma^{\perp}$. Since $u \in B V(\Omega)$ and $S$ is composed by a finite number of points, we deduce $|D u|(S)=0$ which implies $|\mu|(S)=0$. Hence $\sigma$ writes in the form $U\left(M_{\sigma}, \theta_{\sigma}, \xi_{\sigma}\right)$.

Let us now use the local results of Section 3 to prove the $\Gamma-\lim$ inf inequality.

Proof of Theorem 1.1. Let $\left(\sigma_{\varepsilon}, \phi_{\varepsilon}\right)$ such that $\sigma_{\varepsilon} \stackrel{*}{\rightarrow} \sigma$ and $\phi_{\varepsilon} \rightarrow \phi$ as in the statement of the theorem. Without loss of generality, we can suppose that $\mathcal{F}_{\varepsilon}\left(\sigma_{\varepsilon}, \phi_{\varepsilon}\right)<+\infty$.

Let $\hat{\Omega}$ be as in the proof of Theorem 1.3 and let us define $\mu=\Gamma-\liminf _{\varepsilon} \mathcal{F}_{\varepsilon}\left(\sigma_{\varepsilon}, \phi_{\varepsilon}\right)$ and $\lambda=\beta|\sigma|+\mathcal{H}^{1}\left\llcorner M_{\sigma}\right.$. Consider the countable family of sets $\left\{O_{i}\right\} \subset \mathcal{A}_{S}(\hat{\Omega})$ made of the relatively open rectangles $O_{i} \subset \hat{\Omega} \backslash S$ with vertices in $\mathbf{Q}^{2}$. The local result stated in Theorem 3.1 gives for any $i \in \mathbf{N}$

$$
\mu(A) \geq \mu\left(O_{i} \cap A\right) \geq \lambda\left(O_{i} \cap A\right)=\int_{A} \psi_{i} \mathrm{~d} \lambda .
$$

where $\psi_{i}:=1_{O_{i}}$. Therefore Lemma 3.1 gives

$$
\Gamma-\liminf _{\varepsilon \downarrow 0} \mathcal{F}_{\varepsilon}\left(\sigma_{\varepsilon}, \phi_{\varepsilon}\right)=\mu(\hat{\Omega}) \geq \lambda(\hat{\Omega})=\beta|\sigma|(\bar{\Omega})+\mathcal{H}^{1}\left(M_{\sigma}\right)
$$

since $\sup _{i} \psi_{i}$ is the constant function 1 .

\section{5. $\Gamma$-limsup inequality}

Let us prove the $\Gamma$-limsup inequality stated in Theorem 1.2. Recall that the latter consists in finding a sequence $\left(\sigma_{\varepsilon}, \phi_{\varepsilon}\right)$ for any given couple $(\sigma, \phi) \in \mathcal{M}\left(\bar{\Omega}, \mathbf{R}^{2}\right) \times L^{1}(\Omega)$ such that $\sigma_{\varepsilon} \stackrel{*}{\rightarrow} \sigma, \phi_{\varepsilon} \rightarrow \phi$ in $L^{1}(\Omega)$ and

$$
\limsup _{\varepsilon \downarrow 0} \mathcal{F}_{\varepsilon}\left(\sigma_{\varepsilon}, \phi_{\varepsilon}\right) \leq \mathcal{E}_{\beta}(\sigma, \phi)
$$

When $\mathcal{E}_{\beta}(\sigma, \phi)=+\infty$ the inequality is valid for any sequence therefore by definition (1.5) we can assume $\sigma=U(M, \theta, \xi)$ and $\phi=1$. In view of the results from White [18], [19] and 
Xia [20] polyhedral vector measures are dense in energy and it is sufficient to consider vector measures of the form

$$
\sigma=\sum_{i=1}^{n} U\left(M_{i}, \theta_{i}, \xi_{i}\right)
$$

where $M_{i}$ is a segment, $\theta_{i} \in \mathbf{R}_{+}$is $\mathcal{H}^{1}$-a.e. constant and $\xi_{i}$ is an orientation of $M_{i}$ for each $i$. An exaustive proof of the same result in the general case can be found in the recent paper [11]. Nevertheless, to keep self-contained our work, we included in appendix A a proof of this result based on BV functions. Without loss of generality we can suppose that for each couple of segments $M_{i}, M_{j}$, for $i \neq j$, the intersection $M_{i} \cap M_{j}$ is at most a point (called branching point) not belonging to the relative interior of $M_{i}$ and $M_{j}$. We first produce the estimate (5.1) for $\sigma$ concentrated on a single segment thus let us assume $\sigma=\theta e_{1} \cdot \mathcal{H}^{1}\llcorner(0, l) \times\{0\}$.

Notation: Let us fix the values

$$
a_{\varepsilon}:=\left\{\begin{array}{cc}
\frac{\theta \beta \varepsilon}{2} & \text { if } \beta>0 \\
\varepsilon & \text { if } \beta=0
\end{array}, \quad b_{\varepsilon}:=\varepsilon \ln \left(\frac{1-\eta}{\varepsilon}\right) \quad \text { and } \quad r_{\varepsilon}=\max \left\{\varepsilon, a_{\varepsilon}\right\} .\right.
$$

Let $d_{\infty}(x, S)$ be the distance function from $x$ to the set $S \subset \Omega$ relative to the infinity norm on $\mathbf{R}^{2}$ and $Q_{r}(P)=\left\{x \in \mathbf{R}^{2}: d_{\infty}(x, P) \leq r\right\}$ the square centered in $P$ of size $2 r$ and sides parallel to the axes. Introduce the sets

$$
\begin{aligned}
I_{a_{\varepsilon}} & :=\left\{x \in \mathbf{R}^{2}: d_{\infty}(x,[0, l] \times\{0\}) \leq a_{\varepsilon}\right\} \cup Q_{r_{\varepsilon}}(0,0) \cup Q_{r_{\varepsilon}}(l, 0), \\
I_{b_{\varepsilon}} & :=\left\{x \in \mathbf{R}^{2}: d_{\infty}\left(x, I_{a_{\varepsilon}}\right) \leq b_{\varepsilon}\right\}, \\
I_{c_{\varepsilon}} & :=\left\{x \in \mathbf{R}^{2}: d_{\infty}\left(x,\left(I_{a_{\varepsilon}} \cup I_{b_{\varepsilon}}\right)\right) \leq \varepsilon\right\}, \\
I_{d_{\varepsilon}} & :=\Omega \backslash\left(I_{a_{\varepsilon}} \cup I_{b_{\varepsilon}} \cup I_{c_{\varepsilon}}\right), \\
\Sigma_{\varepsilon}(t) & :=\left\{\left(t, x_{2}\right):\left|x_{2}\right| \leq r_{\varepsilon}\right\},
\end{aligned}
$$

and define $R_{\varepsilon}=I_{a_{\varepsilon}} \backslash\left(Q_{r_{\varepsilon}}(0,0) \cup Q_{r_{\varepsilon}}(l, 0)\right)$.
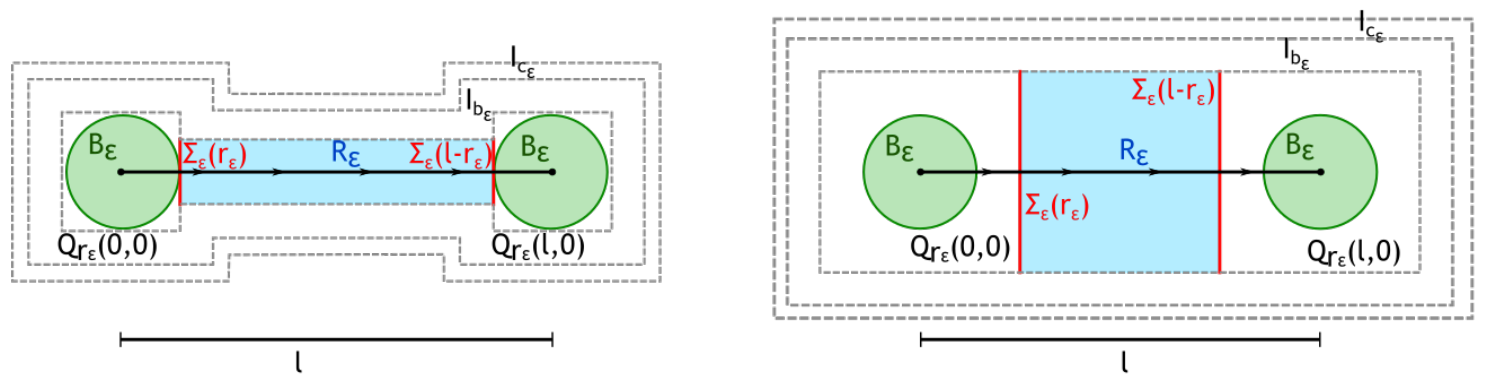

Figure 2: Example of the neighborhoods of the segment $[0, l] \times\{0\}$. On the left the case in which $r_{\varepsilon}=\varepsilon$, on the right the case in which $r_{\varepsilon}=a_{\varepsilon}>\varepsilon$. The cyan region is $R_{\varepsilon}$ and $I_{a_{\varepsilon}}=R_{\varepsilon} \cup\left(Q_{r_{\varepsilon}}(0,0) \cup Q_{r_{\varepsilon}}(l, 0)\right)$. Remark that $\operatorname{supp}\left(\rho_{\varepsilon}\right)=B(0, \varepsilon)$.

Costruction of $\sigma_{\varepsilon}$ : We build $\sigma_{\varepsilon}$ as a vector field supported on $I_{a_{\varepsilon}}$. In particular we add together three different constructions performed respectively on $R_{\varepsilon}, Q_{r_{\varepsilon}}(0,0)$ and 
$Q_{r_{\varepsilon}}(l, 0)$. We construct $\sigma_{\varepsilon}$ on $R_{\varepsilon}$ in order to obtain the $\Gamma$-limsup inequality, on the other hand we are forced to modify such construction in a square neighborhood of each ending point of the segment to control $\nabla \cdot \sigma_{\varepsilon}$. As a matter of fact we need to verify that the piecewise definition coincide on the sets $\Sigma_{\varepsilon}\left(r_{\varepsilon}\right)$ and $\Sigma_{\varepsilon}\left(l-r_{\varepsilon}\right)$ which correspond to the interfaces between $Q_{r_{\varepsilon}}(0,0)$ and $R_{\varepsilon}$, and, $R_{\varepsilon}$ and $Q_{r_{\varepsilon}}(l, 0)$. Let $r=r_{\varepsilon} / \varepsilon$ and consider the problem

$$
\begin{cases}\Delta u= \pm \theta\left(\delta_{x_{0}} * \rho\right), & \text { on } Q_{r}(0,0) \\ \frac{\partial u}{\partial \nu}=\frac{ \pm \theta}{\mathcal{H}^{1}(\Sigma)}, & \text { on } \Sigma^{ \pm}=\left\{x \in \mathbf{R}^{2}: x_{1}= \pm 1,\left|x_{2}\right| \leq \frac{\theta \beta}{2}\right\} \\ \frac{\partial u}{\partial \nu}=0, & \text { otherwise on } \partial Q_{r}(0,0) \backslash \Sigma^{ \pm} .\end{cases}
$$

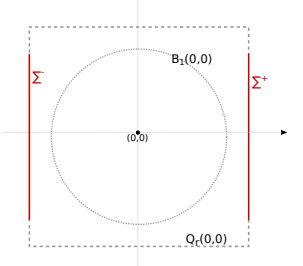

In the latter the set $\Sigma^{+}\left(\right.$resp. $\left.\Sigma^{-}\right)$is the image of the set $\Sigma_{\varepsilon}\left(r_{\varepsilon}\right)\left(\right.$ resp. $\left.\Sigma_{\varepsilon}\left(l-r_{\varepsilon}\right)\right)$ via the map

$$
x \longmapsto \frac{x}{\varepsilon}, \quad\left(\operatorname{resp.} \quad x \longmapsto \frac{x-(l, 0)}{\varepsilon}\right) .
$$

Let $u^{+}$be the solution relative to the problem in which every occurrence of \pm is replaced by + and let $u^{-}$defined accordingly. Then set

$$
\sigma_{\varepsilon}= \begin{cases}\frac{\nabla u^{+}(x / \varepsilon)}{\varepsilon}, & \text { on } Q_{r_{\varepsilon}}(0,0), \\ \frac{\theta}{2 a_{\varepsilon}} \cdot e_{1}, & \text { on } R_{\varepsilon} \\ \frac{\nabla u^{-}((x-(l, 0)) / \varepsilon)}{\varepsilon}, & \text { on } Q_{r_{\varepsilon}}(l, 0)\end{cases}
$$

Indeed, the Neumann Boundary conditions imposed for $u^{+}\left(\operatorname{resp} . u^{-}\right)$on $\Sigma^{+}\left(\operatorname{resp} . \Sigma^{-}\right)$ ensure that the latter piecewise definition is continuous on $\Sigma_{\varepsilon}\left(r_{\varepsilon}\right)$ and $\Sigma_{\varepsilon}\left(l-r_{\varepsilon}\right)$. By construction we have that $\nabla \cdot \sigma_{\varepsilon}=\theta\left[\left(\delta_{(0,0)}-\delta_{(l, 0)}\right) * \rho_{\varepsilon}\right]$ and $\sigma_{\varepsilon} \stackrel{*}{\rightarrow} \sigma$. Let us point out as well that there exists a constant $c(\alpha, \theta)$ such that

$c(\alpha, \theta):=\int_{Q_{r_{\varepsilon}}(l, 0)}\left|\sigma_{\varepsilon}\right|^{2} \mathrm{~d} x=\int_{Q_{r_{\varepsilon}}(0,0)}\left|\sigma_{\varepsilon}\right|^{2} \mathrm{~d} x=\int_{Q_{r}(0,0)}\left|\nabla u^{+}(x)\right|^{2} \mathrm{~d} x=\int_{Q_{r}(0,0)}\left|\nabla u^{-}(x)\right|^{2} \mathrm{~d} x$.

Costruction of $\phi_{\varepsilon}$ : Most of the properties of $\phi_{\varepsilon}$ are a consequence of the inequalities obtained in Theorem 3.1 and the structure of $\sigma_{\varepsilon}$. On one hand we need $\phi_{\varepsilon}$ to attain the lowest value possible on $I_{a_{\varepsilon}}$ in order to compensate the concentration of $\sigma_{\varepsilon}$ in this set, on the other, as shown in inequality (3.8), we need to provide the optimal profile for the transition from this low value to 1 . For this reasons we are led to consider the following ordinary differential equation associated with the optimal transition

$$
\left\{\begin{array}{l}
w_{\varepsilon}^{\prime}=\frac{1}{\varepsilon}\left(1-w_{\varepsilon}\right), \\
w_{\varepsilon}(0)=\eta
\end{array}\right.
$$


Observe that $w_{\varepsilon}=1-(1-\eta) \exp \left(\frac{-t}{\varepsilon}\right)$ is the explicit solution of equation (5.5) and set

$$
\phi_{\varepsilon}(x):= \begin{cases}\eta, & \text { if } x \in I_{a_{\varepsilon}}, \\ w_{\varepsilon}\left(d_{\infty}\left(x, I_{a_{\varepsilon}}\right)\right), & \text { if } x \in I_{b_{\varepsilon}}, \\ d_{\infty}\left(x, I_{b_{\varepsilon}}\right)-\varepsilon+1, & \text { if } x \in I_{c_{\varepsilon}} \\ 1, & \text { otherwise }\end{cases}
$$

Evaluation of $\mathcal{F}_{\varepsilon}\left(\sigma_{\varepsilon}, \phi_{\varepsilon}\right)$ : (case of a $\sigma$ concentrated on a line segment) We prove inequality (5.1) for the sequence we have produced. Since the sets $I_{a_{\varepsilon}}, I_{b_{\varepsilon}}, I_{c_{\varepsilon}}$ and $I_{d_{\varepsilon}}$ are disjoint we can split the energy as follows

$$
\mathcal{F}_{\varepsilon}\left(\sigma_{\varepsilon}, \phi_{\varepsilon}\right)=\mathcal{F}_{\varepsilon}\left(\sigma_{\varepsilon}, \phi_{\varepsilon} ; I_{a_{\varepsilon}}\right)+\mathcal{F}_{\varepsilon}\left(\sigma_{\varepsilon}, \phi_{\varepsilon} ; I_{b_{\varepsilon}}\right)+\mathcal{F}_{\varepsilon}\left(\sigma_{\varepsilon}, \phi_{\varepsilon} ; I_{c_{\varepsilon}}\right)+\mathcal{F}_{\varepsilon}\left(\sigma_{\varepsilon}, \phi_{\varepsilon} ; I_{d_{\varepsilon}}\right)
$$

and evaluate each component individually. Since $\sigma_{\varepsilon}$ is null and $\phi_{\varepsilon}$ is constant and equal to 1 in $I_{d_{\varepsilon}}$ we have that $\mathcal{F}_{\varepsilon}\left(\sigma, \phi_{\varepsilon} ; I_{d_{\varepsilon}}\right)=0$. For the other components we strongly use the definitions in (5.3) and (5.6). First we split again the energy on the set $I_{a_{\varepsilon}}$ as following

$$
\mathcal{F}_{\varepsilon}\left(\sigma_{\varepsilon}, \phi_{\varepsilon} ; I_{a_{\varepsilon}}\right)=\mathcal{F}_{\varepsilon}\left(\sigma_{\varepsilon}, \phi_{\varepsilon} ; R_{\varepsilon}\right)+\mathcal{F}_{\varepsilon}\left(\sigma_{\varepsilon}, \phi_{\varepsilon} ; Q_{r_{\varepsilon}}(0,0)\right)+\mathcal{F}_{\varepsilon}\left(\sigma_{\varepsilon}, \phi_{\varepsilon} ; Q_{r_{\varepsilon}}(l, 0)\right) .
$$

Now identity (5.4) leads to the estimate

$$
\mathcal{F}_{\varepsilon}\left(\sigma_{\varepsilon}, \phi_{\varepsilon} ; Q_{r_{\varepsilon}}(0,0)\right)=\mathcal{F}_{\varepsilon}\left(\sigma_{\varepsilon}, \phi_{\varepsilon} ; Q_{r_{\varepsilon}}(l, 0)\right)=\frac{\eta^{2}}{2 \varepsilon} c(\beta, \theta)+\frac{(1-\eta)^{2}}{2 \varepsilon} r_{\varepsilon}^{2}
$$

and

$$
\mathcal{F}_{\varepsilon}\left(\sigma_{\varepsilon}, \phi_{\varepsilon} ; R_{\varepsilon}\right)=\left[\frac{1}{2 \varepsilon} \eta^{2}\left|\frac{\theta}{2 a_{\varepsilon}}\right|^{2}+\frac{(1-\eta)^{2}}{2 \varepsilon}\right]\left|R_{\varepsilon}\right| \leq\left[\frac{(\theta \eta)^{2}}{8 \varepsilon a_{\varepsilon}^{2}}+\frac{1}{2 \varepsilon}\right] 2 a_{\varepsilon} l .
$$

Then passing to the limsup we obtain

$$
\limsup _{\varepsilon \downarrow 0} \mathcal{F}_{\varepsilon}\left(\sigma_{\varepsilon}, \phi_{\varepsilon} ; I_{a_{\varepsilon}}\right) \leq \theta \beta l=\theta \beta \mathcal{H}^{1}([0, l] \times\{0\})
$$

To obtain the inequality on the sets $I_{b_{\varepsilon}}$ and $I_{c_{\varepsilon}}$ we are going to apply the coarea formula therefore let us observe that for both $d_{\infty}\left(x, I_{a_{\varepsilon}}\right)$ and $d_{\infty}\left(x, I_{b_{\varepsilon}}\right)$ there holds $\left|\nabla d_{\infty}(x, \cdot)\right|=1$ for a.e. $x \in \Omega$ and that there exist a constant $k=k(\beta, \theta)$ such that the level lines $\left\{d_{\infty}(x, \cdot)=t\right\}$ have $\mathcal{H}^{1}$ length controlled by $2 l+k t$. In view of these remarks we obtain

$$
\begin{aligned}
\mathcal{F}_{\varepsilon}\left(\sigma_{\varepsilon}, \phi_{\varepsilon} ; I_{b_{\varepsilon}}\right) & =\int_{I_{b_{\varepsilon}}}\left[\frac{\varepsilon}{2}\left|\nabla \phi_{\varepsilon}\right|^{2}+\frac{\left(1-\phi_{\varepsilon}\right)^{2}}{2 \varepsilon}\right]\left|\nabla d_{\infty}\left(x, I_{a_{\varepsilon}}\right)\right| \mathrm{d} x \\
& =\int_{0}^{b_{\varepsilon}}\left[\frac{\left(1-w_{\varepsilon}(t)\right)^{2}}{2 \varepsilon}+\frac{\varepsilon}{2}\left|w_{\varepsilon}^{\prime}(t)\right|^{2}\right] \mathcal{H}^{1}\left(\left\{d_{\infty}\left(\cdot, I_{a_{\varepsilon}}\right)=t\right\}\right) \mathrm{d} t \\
& \leq(2 l+k \varepsilon)\left[\frac{1}{2}\left(1-w_{\varepsilon}(t)\right)^{2}\right]_{0}^{b_{\varepsilon}} \\
& =\left(l-\frac{k \varepsilon}{2}\right)\left[(1-\eta)^{2}-\varepsilon^{2}\right] \underset{\varepsilon \downarrow 0}{\longrightarrow} l=\mathcal{H}^{1}([0, l] \times\{0\})
\end{aligned}
$$


and

$$
\begin{aligned}
\mathcal{F}_{\varepsilon}\left(\sigma_{\varepsilon}, \phi_{\varepsilon} ; I_{c_{\varepsilon}}\right) & =\int_{I_{c_{\varepsilon}}}\left[\frac{\varepsilon}{2}\left|\nabla \phi_{\varepsilon}\right|^{2}+\frac{\left(1-\phi_{\varepsilon}\right)^{2}}{2 \varepsilon}\right]\left|\nabla d_{\infty}\left(x, I_{b_{\varepsilon}}\right)\right| \mathrm{d} x \\
& =\int_{0}^{\varepsilon}\left[\frac{(1-t+\varepsilon-1)^{2}}{2 \varepsilon}+\frac{\varepsilon}{2}\right] \mathcal{H}^{1}\left(\left\{d_{\infty}\left(\cdot, I_{b_{\varepsilon}} \cup I_{a_{\varepsilon}}\right)=t\right\}\right) \mathrm{d} t \\
& \leq(2 l+k \varepsilon)\left[\frac{(t-\varepsilon)^{3}}{6 \varepsilon}+\frac{\varepsilon}{2} t\right]_{0}^{\varepsilon} \\
& =(2 l+k \varepsilon) \varepsilon^{2} \frac{2}{3} \underset{\varepsilon \downarrow 0}{\longrightarrow} 0 .
\end{aligned}
$$

Finally adding up equations (5.7), (5.8), (5.9) and (5.10) we obtain

$$
\limsup _{\varepsilon \downarrow 0} \mathcal{F}_{\varepsilon}\left(\sigma_{\varepsilon}, \phi_{\varepsilon}\right) \leq(1+\beta \theta) \mathcal{H}^{1}([0, l] \times\{0\}) .
$$

Case of a generic $\sigma$ of the form (5.2):

Let us call $\sigma_{\varepsilon}^{i}, \phi_{\varepsilon}^{i}$ the functions obtained above for each $\sigma_{i}=\theta_{i} \xi_{i} \mathcal{H}^{1}\left\llcorner M_{i}\right.$ and set

$$
\sigma_{\varepsilon}=\sum_{i=1}^{n} \sigma_{\varepsilon}^{i}, \quad \phi_{\varepsilon}=\min _{i} \phi_{\varepsilon}^{i} .
$$

In view of the contraint (1.1), it holds

$$
\left(\omega_{1}-\omega_{2}\right)=\nabla \cdot \sigma=\sum_{i} \nabla \cdot\left(\theta_{i} \xi_{i} \mathcal{H}^{1}\left\llcorner M_{i}\right)=\sum_{i} \theta_{i}\left(\delta_{P_{i}^{+}}-\delta_{P_{i}^{-}}\right),\right.
$$

where $P_{i}^{+}$and $P_{i}^{-}$are the starting and ending point of the segment $M_{i}$ according to its orientation $\xi_{i}$. Replacing each $\sigma_{i}$ with $\sigma_{\varepsilon}^{i}$ we have

$$
\nabla \cdot \sigma_{\varepsilon}=\sum_{i} \nabla \cdot \sigma_{\varepsilon}^{i}=\sum_{i} \theta_{i}\left(\delta_{P_{i}^{+}}-\delta_{P_{i}^{-}}\right) * \rho_{\varepsilon}=\left(\omega_{1}-\omega_{2}\right) * \rho_{\varepsilon} .
$$

Thus $\sigma_{\varepsilon}$ satisfies constraint (1.2). We now prove inequality (5.1). The following inequality holds true

$$
\begin{aligned}
\mathcal{F}_{\varepsilon}\left(\sigma_{\varepsilon}, \phi_{\varepsilon}\right) & =\int_{\Omega} \frac{1}{2 \varepsilon}\left|\min _{i} \phi_{\varepsilon}^{i}\right|^{2}\left|\sum_{i=1}^{n} \sigma_{\varepsilon}^{i}\right|^{2}+\frac{\varepsilon}{2}\left|\nabla\left(\min _{i} \phi_{\varepsilon}^{i}\right)\right|^{2}+\frac{\left(1-\min _{i} \phi_{\varepsilon}^{i}\right)^{2}}{2 \varepsilon} \mathrm{d} x \\
& \leq \int_{\Omega} \frac{1}{2 \varepsilon}\left|\min _{i} \phi_{\varepsilon}^{i}\right|^{2}\left|\sum_{i=1}^{n} \sigma_{\varepsilon}^{i}\right|^{2} \mathrm{~d} x+\sum_{i=1}^{n} \int_{\Omega} \frac{\varepsilon}{2}\left|\nabla \phi_{\varepsilon}^{i}\right|^{2}+\frac{\left(1-\phi_{\varepsilon}^{i}\right)^{2}}{2 \varepsilon} \mathrm{d} x
\end{aligned}
$$

therefore let us estimate the first integral in the latter. Observe that for $\varepsilon$ sufficiently small we can assume that all the $R_{\varepsilon}^{i}$ are pairwise disjoint thus we study the behavior in the squares. Let $M_{i_{1}}, \ldots, M_{i_{m_{P}}}$ be the segments meeting at a branching point $P$. For $j=i_{1}, \ldots, i_{m_{P}}$ let us call $Q_{r_{\varepsilon}^{j}}(P)$ the squared neighborhood of $P$ relative to the segment $M_{j}$ as constructed previously. Let us recall that by definition $\phi_{\varepsilon}$ is constant and equal to 
$\eta$ on $\cup_{j=i_{1}}^{m_{P}} Q_{r_{\varepsilon}^{j}}(P)$ then we have the estimate

$$
\begin{aligned}
\int_{\cup_{j=i_{1}}^{m_{P}}\left(R_{\varepsilon}^{j} \cup Q_{r_{\varepsilon}^{j}}(P)\right)} \frac{\phi_{\varepsilon}^{2}}{2 \varepsilon}\left|\sigma_{\varepsilon}\right|^{2} \mathrm{~d} x & =\sum_{j=i_{1}}^{m_{P}} \int_{R_{\varepsilon}^{j}} \frac{\phi_{\varepsilon}^{2}}{2 \varepsilon}\left|\sigma_{\varepsilon}\right|^{2} \mathrm{~d} x+\int_{\cup_{i=i_{1}}^{m_{P}} Q_{r_{\varepsilon}^{j}}(P)} \frac{\phi_{\varepsilon}^{2}}{2 \varepsilon}\left|\sum_{j=i_{1}}^{m_{P}} \sigma_{\varepsilon}^{j}\right|^{2} \mathrm{~d} x \\
& \leq \sum_{j=i_{1}}^{m_{P}} \int_{R_{\varepsilon}^{j}} \frac{\phi_{\varepsilon}^{2}}{2 \varepsilon}\left|\sigma_{\varepsilon}\right|^{2} \mathrm{~d} x+m_{P} \frac{\eta^{2}}{2 \varepsilon} \sum_{j=i_{1}}^{m_{P}} \int_{Q_{r_{\varepsilon}^{j}}(P)}\left|\sigma_{\varepsilon}^{j}\right|^{2} \mathrm{~d} x \\
& \leq \sum_{j=i_{1}}^{m_{P}} \int_{\left(R_{\varepsilon}^{j} \cup Q_{r_{\varepsilon}^{j}}(P)\right)} \frac{1}{2 \varepsilon}\left|\phi_{\varepsilon}^{j}\right|^{2}\left|\sigma_{\varepsilon}^{j}\right|^{2} \mathrm{~d} x+\underbrace{\left(m_{P}-1\right)\left(\sum_{j=i_{1}}^{i_{m_{P}}} c\left(\beta, \theta_{j}\right)\right)}_{c\left(m_{P}, \beta, \theta_{i_{1}}, \ldots, \theta_{i_{m_{P}}}\right) \varepsilon} \frac{\eta^{2}}{2 \varepsilon} .
\end{aligned}
$$

Applying inequality (5.12) on each branching point in equation (5.11) and recomposing the integral gives

$$
\begin{aligned}
\limsup _{\varepsilon \downarrow 0} \mathcal{F}_{\varepsilon}\left(\sigma_{\varepsilon}, \phi_{\varepsilon}\right) & \leq \limsup _{\varepsilon \downarrow 0} \sum_{i=1}^{n} \mathcal{F}_{\varepsilon}\left(\sigma_{\varepsilon}^{i}, \phi_{\varepsilon}^{i}\right)+n c\left(n, \beta, \theta_{i}, \ldots, \theta_{n}\right) \varepsilon \\
& \leq \sum_{i=1}^{n}\left(1+\beta \theta_{i}\right) \mathcal{H}^{1}\left(M_{i}\right) \\
& =\int_{\operatorname{supp}(\sigma)}(1+\beta \theta) \mathrm{d} \mathcal{H}^{1}=\mathcal{E}_{\beta}(\sigma, 1)
\end{aligned}
$$

which ends the proof.

\section{Numerical Approximation}

In this section we present numerical evidence of the $\Gamma$-convergence result we have shown in the setting introduced in Remark 1. The first issue we address is how to impose the divergence constraint. To this aim it is convenient to introduce the following notation

$$
\begin{aligned}
& f_{\varepsilon}=\left(\delta_{x_{0}}-\frac{1}{N} \sum_{j=1}^{N} \delta_{x_{j}}\right) * \rho_{\varepsilon}, \\
& G_{\varepsilon}(\sigma, \phi)=\left\{\begin{array}{l}
\int_{\Omega}\left[\frac{1}{2 \varepsilon}|\phi|^{2}|\sigma|^{2}\right] \mathrm{d} x, \\
+\infty,
\end{array} \quad \text { if } \sigma \in V_{\varepsilon},\right. \text { otherwise in } L^{2}\left(\Omega, \mathbf{R}^{2}\right), \\
& \Lambda_{\varepsilon}(\phi)= \begin{cases}\int_{\Omega}\left[\frac{\varepsilon}{2}|\nabla \phi|^{2}+\frac{\left(1-\phi^{2}\right)}{2 \varepsilon}\right] \mathrm{d} x, & \text { if } \phi \in W_{\varepsilon}, \\
+\infty, & \text { otherwise in } L^{1}(\Omega) .\end{cases}
\end{aligned}
$$

Then let us observe that the following equality holds

$$
\min _{\sigma \in L^{2}\left(\Omega, \mathbf{R}^{2}\right)} G_{\varepsilon}(\sigma, \phi)=\inf _{\sigma \in L^{2}\left(\Omega, \mathbf{R}^{2}\right)}\left\{\sup _{u \in W^{1,2}(\Omega)} \int_{\Omega} \frac{1}{2 \varepsilon}|\phi|^{2}|\sigma|^{2}+u\left(\nabla \cdot \sigma-f_{\varepsilon}\right) \mathrm{d} x\right\} .
$$


By von Neumann's min-max Theorem [4, Thm. 9.7.1] we can exchange inf and sup obtaining for each $\varepsilon>0$ and $\phi \in W_{\varepsilon}$

$$
\begin{aligned}
\min _{\sigma} G_{\varepsilon}(\sigma, \phi) & =\sup _{u} \inf _{\sigma} \int_{\Omega} \frac{1}{2 \varepsilon}|\phi|^{2}|\sigma|^{2}-\left(\langle\nabla u, \sigma\rangle+u f_{\varepsilon}\right) \mathrm{d} x \\
& =-\min _{u} \int_{\Omega} \frac{\varepsilon|\nabla u|^{2}}{2|\phi|^{2}}+u f_{\varepsilon} \mathrm{d} x=-\min _{u} \bar{G}_{\varepsilon}(u, \phi),
\end{aligned}
$$

with $\sigma=\frac{\varepsilon \nabla u}{\phi^{2}}$, this naturally leads to the following alternate minimization problem: given an initial guess $\phi_{0}$ we define

$$
\begin{aligned}
\sigma_{j} & :=\frac{\varepsilon \nabla u_{j}}{\phi_{j}^{2}} \quad \text { where } \quad u_{j}:=\operatorname{argmin} \bar{G}_{\varepsilon}\left(u, \phi_{j}\right), \\
\phi_{j+1} & :=\operatorname{argmin} G_{\varepsilon}\left(\sigma_{j}, \phi\right)+\Lambda_{\varepsilon}(\phi) .
\end{aligned}
$$

This formulation led to Algorithm 1. We define a circular domain $\Omega$ containing the points in $S$ endowed with a uniform mesh and four values $\beta, \varepsilon_{i n}, \varepsilon_{\text {end }}$ and $N_{\text {iter }}$ and a gaussian convolution kernel $\rho_{\varepsilon_{\text {end }}}$ in order to define $f_{\varepsilon}$. We have implemented the algorithm in FREEFEM ++ choosing for the discrete spaces for $u$ and $\phi$ the space of piecewise polynomials of order 1 . To validate Algorithm 1 we tested on the constraint

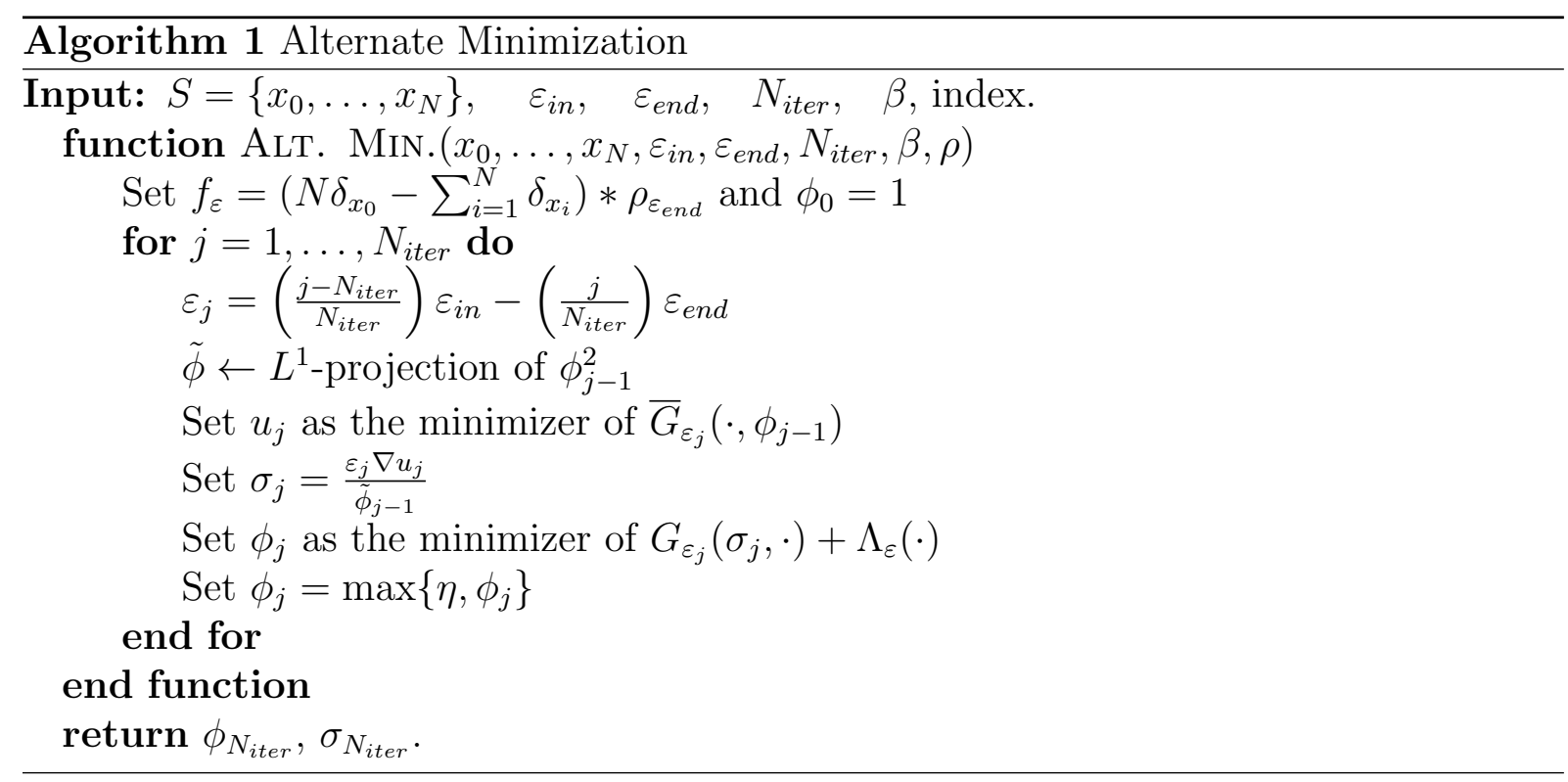

given by four points defining a square inscribed in the unitary circumference, namely $x_{0}=$ $(-\sqrt{2} / 2,-\sqrt{2} / 2), x_{1}=(\sqrt{2} / 2,-\sqrt{2} / 2), x_{2}=(\sqrt{2} / 2, \sqrt{2} / 2)$ and $x_{4}=(-\sqrt{2} / 2, \sqrt{2} / 2)$. Indeed, for such constraint, we can obtain an explicit solution which allows a visual comparison with the one obtained with Algorithm 1. As it is shown in Figure 3 the solution is far from being satisfactory. We think the failure of this procedure is due to the relation between the geometry of the space and the one of the solution itself. In particular, to obtain a good approximation it is necessary to refine the mesh where $\phi$ attains values close to zero but we observed that this restrains the process of approaching the solution. To overcame the problem we propose a modifications to Algorithm (1) to include a step of joint minimization. Let us consider a smooth diffeomorphism $T: \Omega \rightarrow \Omega$ and define

$$
\phi_{T}=\phi \circ T(x), \quad \sigma_{T}=\sigma \circ T(x),
$$



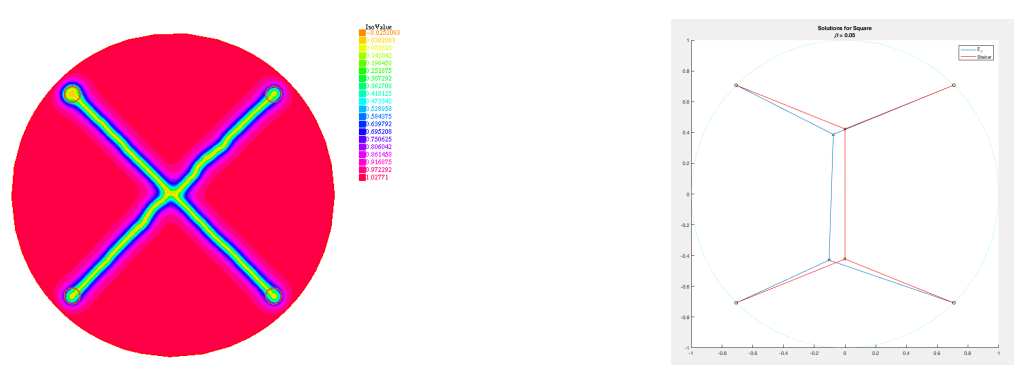

Figure 3: On the left: Graph of $\phi_{\text {Niter }}$ obtained via the Alternate Minimization Algorithm 1. On the right: in red, one of the solutions to the Steiner problem, while in blue, a minimizer of the energy $\mathcal{E}_{\beta}$.

and the functional

$$
F_{\varepsilon}(T)=G_{\varepsilon}(\sigma \circ T, \phi \circ T)+\Lambda_{\varepsilon}(\phi \circ T) .
$$

Let $d F_{\varepsilon}(I d)$ be the differential of the the functional $F_{\varepsilon}$ evaluated for $T=I d$. We represent $d F$ as function $V \in W^{1,2}(\Omega, \Omega)$ by solving the elliptic problem

$$
\langle V, W\rangle_{W^{1,2}}=\left\langle d F_{\varepsilon}(I d), W\right\rangle_{W^{1,2}} \text { for any test vector field } W .
$$

Let $\bar{V}$ be a solution to the latter problem, we perform a gradient descent in the direction $-\bar{V}$. In Algorithm 2, we implemented this joint minimization step. As it is possible to

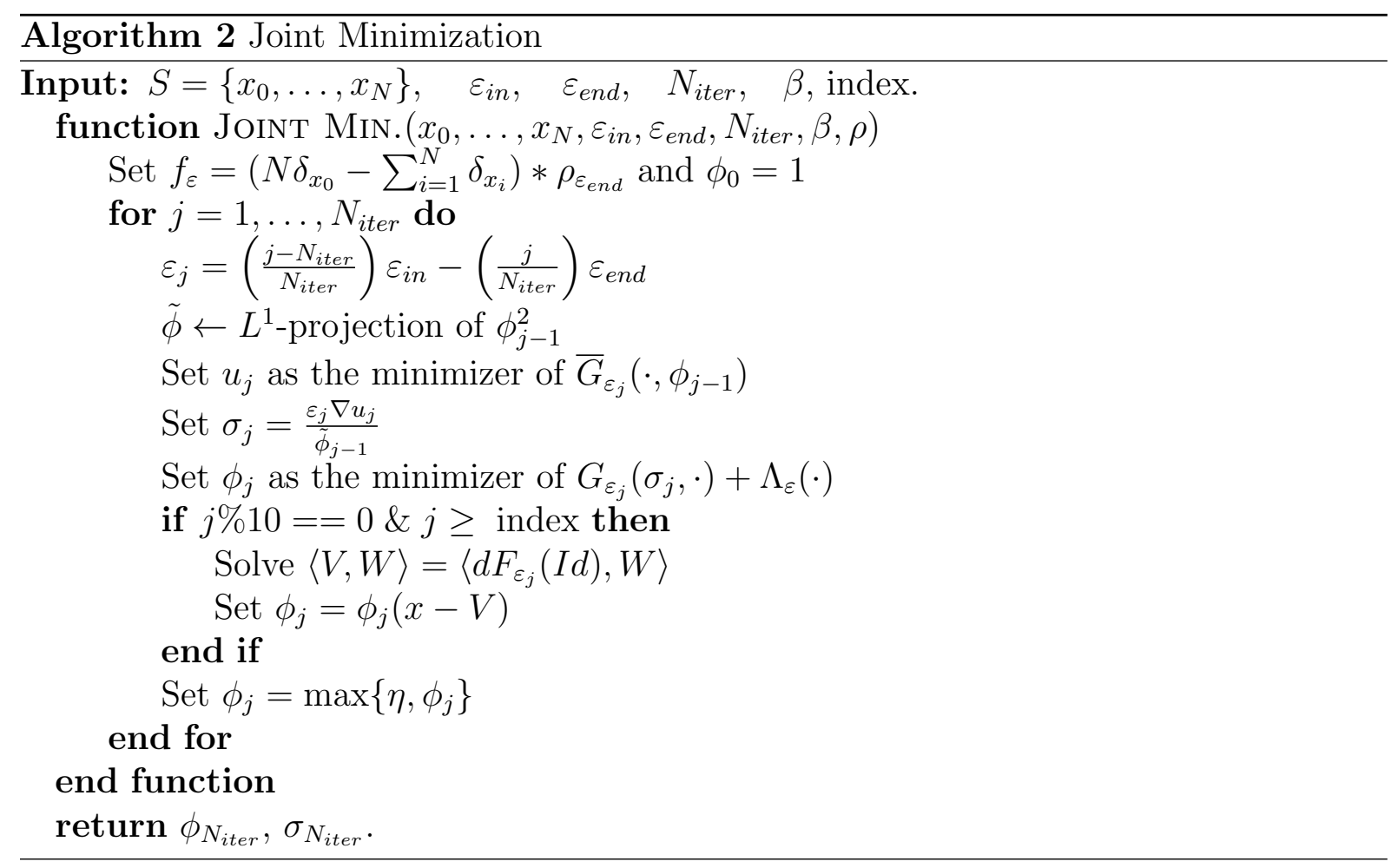

remark from a visual comparison of Figure 4 and Figure 3 the joint minimization procedure allows to displace the functions. Indeed, as shown in Figure 5, the energy decreases during the joint minimization procedure as it. Let us propose a second modification to Algorithm 1. Let us observe that the optimization of the component $\Lambda_{\varepsilon}$ defined in equation (6.1) is the one responsible for the length minimization of the support of $\sigma$. Therefore 

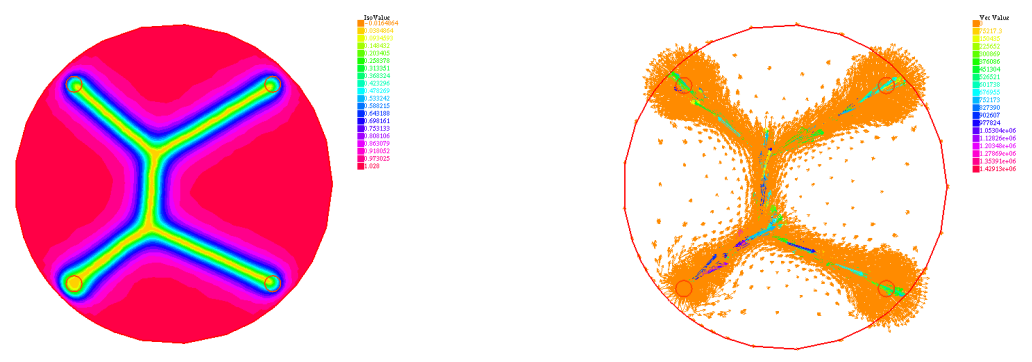

Figure 4: On the left the graph of $\phi_{N i t e r}$ on the right the one of $\sigma_{N i t e r}$ obtained via the Joint Minimization Algorithm 2.

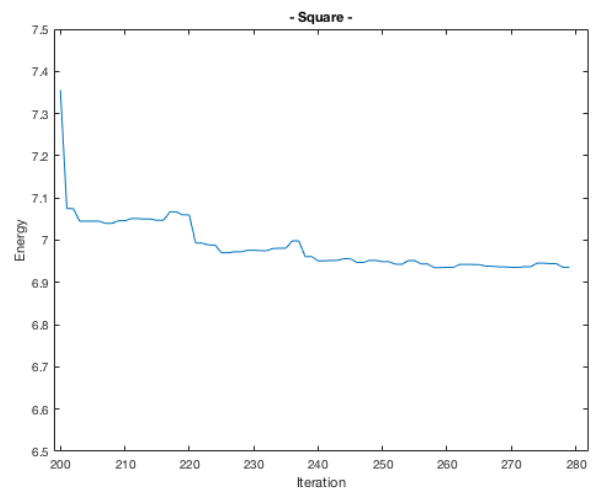

Figure 5: Behaviour of the energy during the joint minimization iterations of Algorithm 2.

it is reasonable to look for a gradient descent in the component $\Lambda_{\varepsilon}$. In Algorithm 3 we implement such procedure. This method enhances the length minimization process since but has the drawback is that displacing $\phi$ and $\sigma$ in the direction $-d \Lambda$ we could loose the divergence constraint. To avoid such eventuality we perform several steps of Alternate Minimization after the displacement. In the next figures we show the graphs obtained for the couple $\left(\sigma_{N_{\text {iter }}}, \phi_{N_{\text {iter }}}\right)$ via Algorithm 3 with the choices $\beta=0.05, \varepsilon_{\text {in }}=0.5, \varepsilon_{\text {end }}=0.05$, $\beta=0.05, N_{\text {iter }}=500$ and index $=300$. We have chosen to make simulations for points located on the vertices of regular polygons of respectively 3, 4, 5 and 6 vertices. A direct visual comparison between the obtained results in Figure 6 and the exact solutions in Figure 7. 

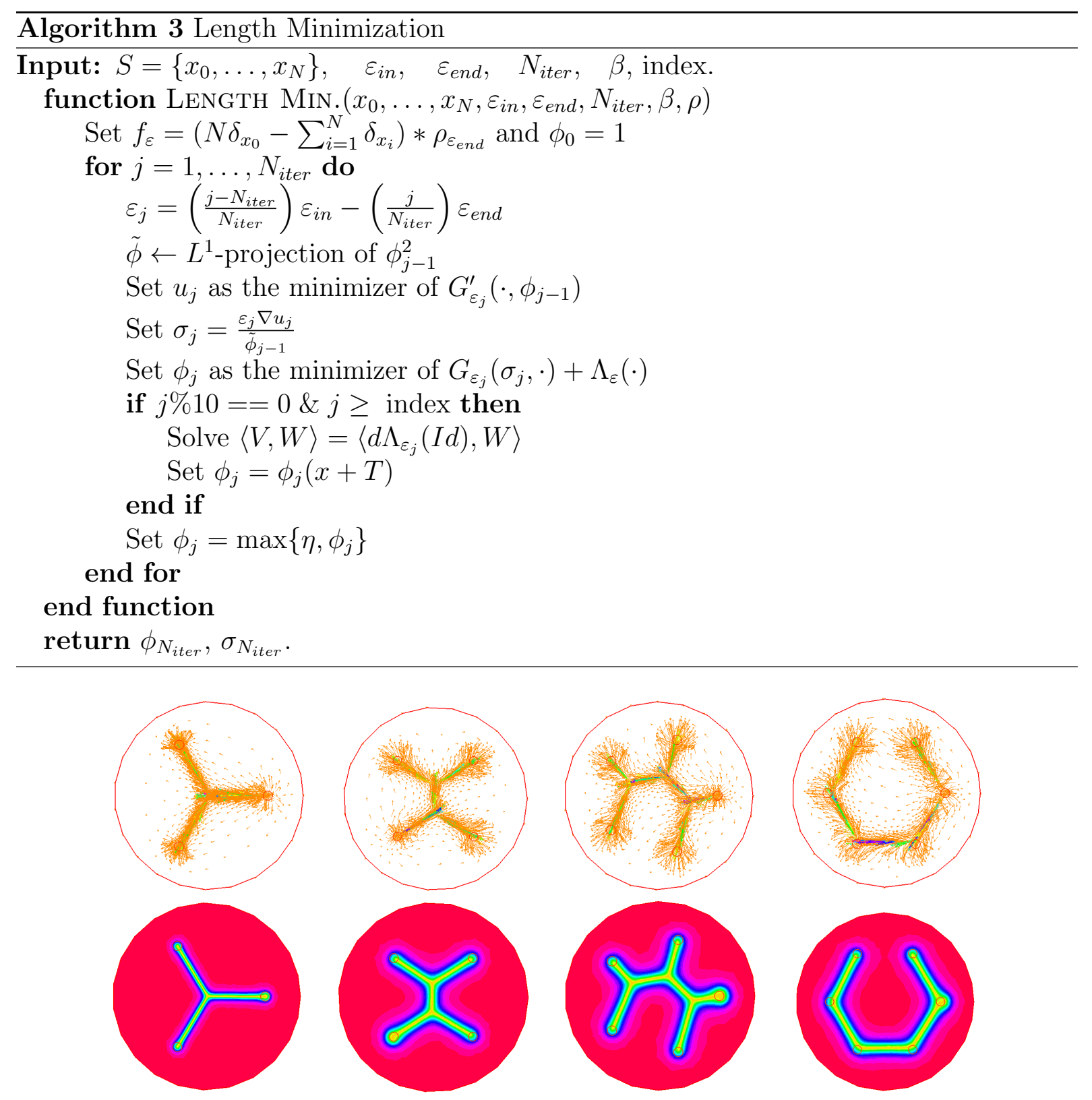

Figure 6: Graph of the couple $\left(\sigma_{N_{i t e r}}, \phi_{N_{i t e r}}\right)$ obtained via Algorithm 1 in the case of 3, 4, 5 and 6 points located on the vertices of a regular polygon.

\section{A. A density result}

We show that measures which have support contained in a finite union of segments, are dense in energy. Without loss of generality let us assume that $\sigma \in \mathcal{M}_{S}(\bar{\Omega})$ is such that $\mathcal{E}_{\beta}(\sigma, 1)<\infty$. In particular $\sigma=U\left(M_{\sigma}, \theta_{\sigma}, \xi_{\sigma}\right)$ is a $\mathcal{H}^{1}$-rectifiable measure. Applying Lemma 4.1 we obtain an $\mathcal{H}^{1}$-rectifiable measure $\gamma=U\left(M_{\gamma}, \theta_{\gamma}, \xi_{\gamma}\right)$ and a partition of $\Omega$ made of polyhedrons $\left\{\Omega_{i}\right\}$ such that $M_{\gamma} \subset \cup_{i} \partial \Omega_{i}, \mathcal{H}^{1}\left(M_{\sigma} \cap \cup_{i} \partial \Omega_{i}\right)=0$ and $\sigma+\gamma$ is divergence free.

From the above properties, we can write

$$
\sigma^{\perp}+\gamma^{\perp}=D u
$$



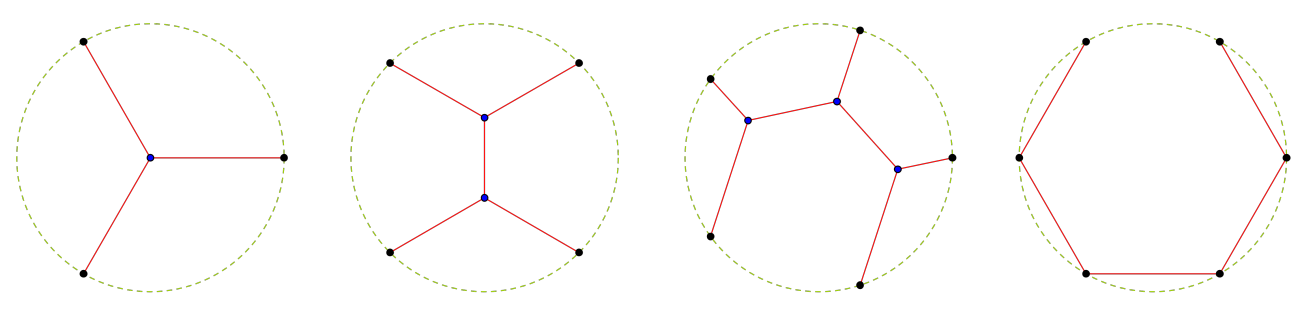

Figure 7: Graph of the exact solutions to the Steiner Problem constrained as in the previous figure.

for some $u \in P C(\Omega)$. Our strategy is the following, using existing results [5], we build an approximating sequence for $u$ on each $\Omega_{j}$ whose gradient is supported on a finite union of segments. We then glue these approximations together to obtain a sequence $\left(w_{j}\right)$ approximating $u$ in $\hat{\Omega}$. The main difficulty is to establish that $D w_{j}\left\llcorner\left[\cup_{i} \partial \Omega_{i}\right]\right.$ is close to $D u\left\llcorner\left[\cup_{i} \partial \Omega_{i}\right]=\gamma^{\perp}\right.$. First let us recall the result in [5]

Lemma A.1. Let $u \in P C(\Omega)$ be such that

$$
\mathcal{E}_{f}(u, \Omega)=\int_{\Omega \cap J_{u}} f([u]) \mathrm{d} \mathcal{H}^{n-1}<+\infty .
$$

for $f$ a continuous, sub-additive and increasing function on $[0,+\infty)$ such that $f(0)=0$ and $\lim _{t \rightarrow 0} \frac{f(t)}{t}=+\infty$. Then there exists a sequence $\left(u_{h}\right) \subset P C(\Omega)$ with the following properties:

- $\lim _{h \rightarrow+\infty} u_{h}=u$ in $L^{1}(\Omega)$,

- $\lim _{h \rightarrow+\infty} \mathcal{E}_{f}\left(u_{h}, \Omega\right)=\mathcal{E}_{f}(u, \Omega)$,

- $J_{u_{h}}$ is contained in a finite union of facets of polytopes for any $h \in \mathbf{N}$. In particular for any $h \in \mathbf{N}$,

$$
\mathcal{H}^{n-1}\left(\Omega \cap \bar{J}_{u_{h}}\right)=\mathcal{H}^{n-1}\left(\Omega \cap J_{u_{h}}\right) \quad \text { and } \quad \mathcal{H}^{n-1}\left(\bar{J}_{u_{h}}\right)<+\infty .
$$

Lemma A.2 (Approximation of $u$ ). There exists a sequence $\left(w_{j}\right) \subset P C(\hat{\Omega})$ with the following properties:

a) $w_{j} \rightarrow u$ weakly in $B V(\hat{\Omega})$,

b) $\operatorname{supp} w_{j} \subset \bar{\Omega}$,

c) $\lim \sup _{j \rightarrow \infty} \mathcal{E}_{\beta}\left(w_{j}, 1\right) \leq \mathcal{E}_{\beta}(u, 1)$,

d) $J_{w_{j}}$ is contained in a finite union of segments for any $j \in \mathbf{N}$,

e) $\left|D w_{j}-D u\right|\left(\cup \partial \Omega_{i}\right) \rightarrow 0$. 
Proof. Step 1: In order to apply the results of [5], we first need to modify $u$ and the energy. Let us denote the energy density function $f(t)=1+\beta t$ and for $k \geq 0$ and $t \geq 0$ let us introduce the approximation

$$
f_{k}(t):= \begin{cases}\left(2^{k / 2}+\beta 2^{-k / 2}\right) \sqrt{t}, & \text { for } t \leq 2^{-k} \\ f(t), & \text { otherwise. }\end{cases}
$$

We have $0 \leq f_{k} \leq f$ and $f_{k} \equiv f$ on $\left[2^{-k},+\infty\right)$. Notice that $f_{k}$ is continuous, sub-additive

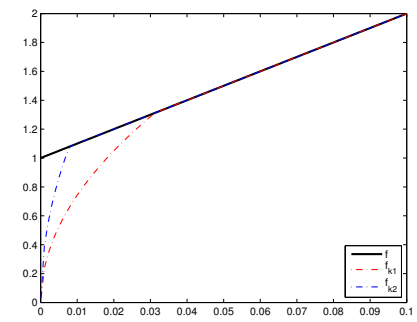

Figure 8: Graph of $\mathrm{f}$ and two of its approximations $f_{k_{1}}$ and $f_{k_{2}}$ with $k_{1}<k_{2}$.

and increasing on $[0,+\infty)$ and that $f_{k}(0)=0$ with $\lim _{t \rightarrow 0} \frac{f_{k}(t)}{t}=+\infty$. We define the associated energy for functions $v \in P C(\hat{\Omega})$ as $\mathcal{E}_{f_{k}}(v, \hat{\Omega}):=\int_{J_{v} \cap \hat{\Omega}} f_{k}([v]) \mathrm{d} \mathcal{H}^{1}$.

Now we denote $P C_{k}(\hat{\Omega})$ the set of functions $v \in P C(\hat{\Omega}),(2.1)$, such that $v(\hat{\Omega}) \subset 2^{-k} \mathbf{Z}$. For these functions we have $\left|v^{+}(x)-v^{-}(x)\right| \geq 2^{-k}$ for $\mathcal{H}^{1}$-almost every $x \in J_{v}$. Consequently, one has

$$
\mathcal{E}_{f_{k}}(v, \hat{\Omega})=\mathcal{E}_{f}(v, \hat{\Omega}) .
$$

For each fixed $k \geq 0$, let us introduce the function

$$
u_{k}=2^{-k}\left\lfloor 2^{k} u\right\rfloor
$$

where $\lfloor t\rfloor$ denotes the integer part of the real $t$. Note that $u_{k} \in P C_{k}(\hat{\Omega})$ with $J_{u_{k}} \subset J_{u}$ and $\left\|u-u_{k}\right\|_{\infty} \leq 2^{-k}$. Notice also that in view of $\left|\left(u_{k}^{+}-u_{k}^{-}\right)-\left(u^{+}-u^{-}\right)\right| \leq 2^{-k}$ we have

$$
\left|D u_{k}-D u\right|(\hat{\Omega}) \leq 2^{-k} \mathcal{H}^{1}\left(J_{u}\right) .
$$

Indeed, $u_{k} \rightarrow u$ strongly in $B V(\hat{\Omega})$, as $\mathcal{H}^{1}\left(J_{u}\right)<+\infty$. Moreover, we see that

$$
\mathcal{E}_{f_{k}}\left(u_{k}, \hat{\Omega}\right)=\mathcal{E}_{f}\left(u_{k}, \hat{\Omega}\right) \leq \mathcal{E}_{f}(u, \hat{\Omega})+\beta 2^{-k} \mathcal{H}^{1}\left(J_{u}\right) .
$$

Step 2: Let us approximate the function $u_{k}$. Let us fix $k \geq 0$ and $\Omega_{i}$. We can apply Lemma A.1 to the function $u_{k}\left\llcorner\Omega_{i}\right.$ and to the energy $\mathcal{E}_{f_{k}}\left(\cdot, \Omega_{i}\right)$. We obtain a sequence $\left(w_{j}^{i}\right)$ which enjoys the following properties:

$w_{j}^{i}\left(\Omega_{i}\right) \subset u_{k}\left(\Omega_{i}\right) \subset 2^{-k} \mathbf{Z}, \quad \forall j \in \mathbf{N}$, hence $w_{j}^{i} \in P C_{k}(\hat{\Omega})$,

$w_{i}^{j} \rightarrow u_{k}$ in $L^{1}\left(\Omega_{i}\right)$ as $j \rightarrow+\infty$,

$\lim _{j \rightarrow+\infty} \mathcal{E}_{f_{k}}\left(w_{j}^{i}, \Omega_{i}\right)=\lim _{j \rightarrow+\infty} \mathcal{E}_{f}\left(w_{j}^{i}, \Omega_{i}\right)=\mathcal{E}_{f}\left(u_{k}, \Omega_{i}\right)$,

$J_{w_{i}^{j}}$ is contained in a finite union of segments for any $j \in \mathbf{N}$,

$\int_{\partial \Omega_{i}}\left|T w_{j}^{i}-T u_{k}\right| \mathrm{d} \mathcal{H}^{1} \rightarrow 0$ where $T: B V\left(\Omega_{i}\right) \rightarrow L^{1}\left(\partial \Omega_{i}\right)$ denotes the trace operator. 
Let us now define globally

$$
w_{j}:=\sum_{j} w_{j}^{i} 1_{\Omega_{i}}
$$

From the above properties, we have $w_{j} \stackrel{*}{\rightarrow} u_{k}$,

$$
\lim \mathcal{E}_{f}\left(w_{j}^{i}, \hat{\Omega}\right)=\mathcal{E}_{f}\left(u_{k}, \Omega_{i}\right)
$$

and

$$
\left|D w_{j}-D u_{k}\right|\left(\cup_{i} \partial \Omega_{i}\right) \rightarrow 0 \quad \text { as } j \rightarrow \infty .
$$

Eventually, using a diagonal argument, we have proved the existence of a sequence $\left(w_{j}\right) \subset$ $P C(\hat{\Omega})$ satisfying claims (a), (b) and (d) of the lemma. Moreover, item (c) is the consequence of (A.2) and (A.3) and item (e) follows from (A.1) and (A.4).

Going back to the $\mathcal{H}^{1}$-rectifiable measures $\sigma=U\left(M_{\sigma}, \theta_{\sigma}, \xi_{\sigma}\right)$, we define the sequence

$$
\sigma_{j}:=-D w_{i}^{\perp}-\gamma
$$

We recall that $\gamma=U\left(M_{\gamma}, \theta_{\gamma}, \xi_{\gamma}\right)$ with $M_{\gamma} \subset \cup \partial \Omega_{i}$. In particular $\gamma=-D u^{\perp}\left\llcorner\left(\cup_{i} \partial \Omega_{i}\right)\right.$. We deduce from the previous lemma:

Lemma A.3. There exists a sequence $\left(\sigma_{j}\right) \in \mathcal{M}_{S}(\bar{\Omega})$ with the properties:

- $\sigma_{j} \rightarrow \sigma$ with respect to weak-* convergence of measures,

- $\sigma_{j}=U\left(M_{\sigma_{j}}, \theta_{\sigma_{j}}, \xi_{\sigma_{j}}\right)$ with $M_{\sigma_{j}}$ contained in a finite union of segments,

- $\lim \sup _{j \rightarrow \infty} \mathcal{E}_{\beta}\left(\sigma_{j}, 1\right) \leq \mathcal{E}_{\beta}(\sigma, 1)$.

\section{Acknowledgments}

The authors have been supported by the ANR project Geometrya, Grant No. ANR-12BS01-0014-01. A.C. also acknowledges the hospitality of Churchill College and DAMTP, U. Cambridge, with a support of the French Embassy in the UK, and a support of the Cantab Capital Institute for Mathematics of Information.

\section{References}

[1] Luigi Ambrosio, Nicola Fusco, and Diego Pallara. Functions of bounded variation and free discontinuity problems. Oxford Mathematical Monographs. The Clarendon Press, Oxford University Press, New York, 2000.

[2] Luigi Ambrosio and Paolo Tilli. Topics on analysis in metric spaces, volume 25 of Oxford Lecture Series in Mathematics and its Applications. Oxford University Press, Oxford, 2004.

[3] Luigi Ambrosio and Vincenzo Maria Tortorelli. Approximation of functionals depending on jumps by elliptic functionals via $\Gamma$-convergence. Comm. Pure Appl. Math., 43(8):999-1036, 1990. 
[4] Hedy Attouch, Giuseppe Buttazzo, and Gérard Michaille. Variational analysis in Sobolev and BV spaces. MOS-SIAM Series on Optimization. Society for Industrial and Applied Mathematics (SIAM), Philadelphia, PA; Mathematical Optimization Society, Philadelphia, PA, second edition, 2014. Applications to PDEs and optimization.

[5] Giovanni Bellettini, Antonin Chambolle, and Michael Goldman. The $\Gamma$-limit for singularly perturbed functionals of Perona-Malik type in arbitrary dimension. Math. Models Methods Appl. Sci., 24(6):1091-1113, 2014.

[6] Marc Bernot, Vicent Caselles, and Jean-Michel Morel. Optimal transportation networks, volume 1955 of Lecture Notes in Mathematics. Springer-Verlag, Berlin, 2009. Models and theory.

[7] Mauro Bonafini, Giandomenico Orlandi, and Edouard Oudet. Variational approximation of functionals defined on 1-dimensional connected sets: the planar case, 2016.

[8] Matthieu Bonnivard, Antoine Lemenant, and Filippo Santambrogio. Approximation of length minimization problems among compact connected sets. SIAM J. Math. Anal., 47(2):1489-1529, 2015.

[9] Andrea Braides. Approximation of free-discontinuity problems, volume 1694 of Lecture Notes in Mathematics. Springer-Verlag, Berlin, 1998.

[10] Andrea Braides. Г-convergence for beginners, volume 22 of Oxford Lecture Series in Mathematics and its Applications. Oxford University Press, Oxford, 2002.

[11] Maria Colombo, Antonio De Rosa, Andrea Marchese, and Salvatore Stuvard. On the lower semicontinuous envelope of functionals defined on polyhedral chains, 2017.

[12] S. Conti, M. Focardi, and F. Iurlano. Phase field approximation of cohesive fracture models. Annales de l'Institut Henri Poincare (C) Non Linear Analysis, pages -, 2015.

[13] E. N. Gilbert and H. O. Pollak. Steiner minimal trees. SIAM J. Appl. Math., 16:1-29, 1968.

[14] Flaviana Iurlano. Fracture and plastic models as $\Gamma$-limits of damage models under different regimes. Adv. Calc. Var., 6(2):165-189, 2013.

[15] Luciano Modica and Stefano Mortola. Un esempio di $\Gamma^{-}$-convergenza. Boll. Un. Mat. Ital. B (5), 14(1):285-299, 1977.

[16] Edouard Oudet and Filippo Santambrogio. A Modica-Mortola approximation for branched transport and applications. Arch. Ration. Mech. Anal., 201(1):115-142, 2011.

[17] Emanuele Paolini and Eugene Stepanov. Existence and regularity results for the Steiner problem. Calc. Var. Partial Differential Equations, 46(3-4):837-860, 2013.

[18] Brian White. The deformation theorem for flat chains. Acta Math., 183(2):255-271, 1999. 
[19] Brian White. Rectifiability of flat chains. Ann. of Math. (2), 150(1):165-184, 1999.

[20] Qinglan Xia. Optimal paths related to transport problems. Commun. Contemp. Math., 5(2):251-279, 2003. 